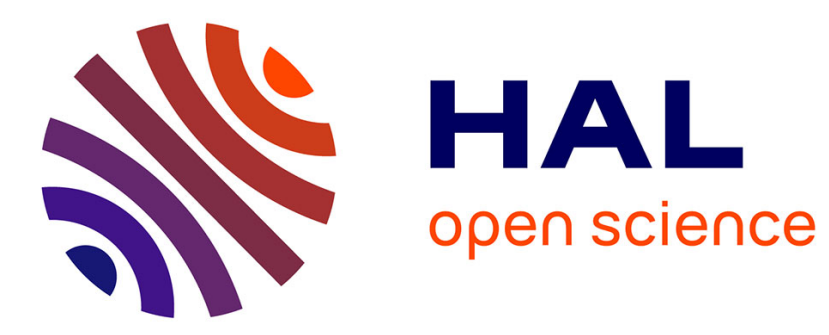

\title{
Les générateurs à plasma
}

\author{
F. Kassabji, Pierre Fauchais
}

\section{- To cite this version:}

F. Kassabji, Pierre Fauchais. Les générateurs à plasma. Revue de Physique Appliquée, 1981, 16 (10), pp.549-577. 10.1051/rphysap:019810016010054900 . jpa-00244949

\section{HAL Id: jpa-00244949 https://hal.science/jpa-00244949}

Submitted on 1 Jan 1981

HAL is a multi-disciplinary open access archive for the deposit and dissemination of scientific research documents, whether they are published or not. The documents may come from teaching and research institutions in France or abroad, or from public or private research centers.
L'archive ouverte pluridisciplinaire HAL, est destinée au dépôt et à la diffusion de documents scientifiques de niveau recherche, publiés ou non, émanant des établissements d'enseignement et de recherche français ou étrangers, des laboratoires publics ou privés. 


\title{
Les générateurs à plasma
}

\author{
F. Kassabji et P. Fauchais \\ Université de Limoges, 123, rue Albert Thomas, 87060 Limoges Cedex, France
}

(Reçu le 6 avril 1981, révisé le 15 juin 1981, accepté le 16 juin 1981)

\begin{abstract}
Résumé. - Cet article présente dans une première partie une étude des phénomènes rencontrés dans les générateurs à plasma. Après une définition sommaire du plasma et de sa génération, nous exposerons les caractéristiques essentielles des arcs plasmas et leurs particularités dans la distribution du potentiel. Le rôle des électrodes apparaît dans les phénomènes d'attachement ainsi que dans les jets cathodique et anodique créés.

Les différentes méthodes de stabilisation de ces arcs (par la paroi, par transpiration, par injection tourbillonnaire d'un liquide ou d'un gaz ou par champ magnétique) sont exposées ainsi que le comportement de l'arc dans son circuit électrique d'alimentation. Les phénomènes propres aux électrodes dans les générateurs à plasma à courant alternatif sont brièvement indiqués et enfin l'usure des électrodes est discutée permettant ainsi d'évaluer la durée de vie des électrodes sous différentes atmosphères.

La seconde partie est consacrée aux principes de conception et de modélisation des générateurs à plasma et la forte influence des propriétés de gaz à haute température. Des relations semi-empiriques permettant la modélisation des différents réacteurs à plasma sont déterminées pour divers gaz.

Dans la troisième partie, et après l'étude des caractéristiques principales des générateurs à plasma à faible enthalpie et de leurs utilisations, nous présentons les divers générateurs à plasma utilisés. La classification faite est liée d'une part à un paramètre de structure : nature des électrodes (chaudes ou froides) et d'autre part à deux paramètres de fonctionnement : mode de stabilisation de la colonne de l'arc et nature des gaz plasmagènes utilisés (oxydants, neutres ou réducteurs).

Enfin, la dernière partie traite des générateurs à plasma à forte enthalpie classés selon les modes de stabilisation utilisés.
\end{abstract}

\begin{abstract}
This paper presents in the first part a study of some phenomena in plasma generators. After a brief definition of the plasma and its production we present the essential characteristics of plasma arcs and their particularities in the potential distribution. The electrodes influence appears in the attachment phenomena as well as in the created cathodic and anodic jets.

The different stabilization methods of these arcs (by wall, by transpiration, by vortex-liquid or gas injection or by magnetic field) are displayed as well as the arc performance in its electric circuit. The specific phenomena of electrodes in alternative current plasma generators are indicated briefly. Finally the electrodes wear is discussed and the life time of electrodes is evaluated under different atmospheres.

The second part is treating of conception and modelling of plasma generators taking into consideration the great influence of high temperature plasma properties. Semi-empirical relations allowing different plasma reactors modelling are determined for different gases.

In the third part, and after the study of essential characteristics of low enthalpy plasma generators and their using, we present the different plasma generators in use. The classification depends on the one hand on a structure parameter : electrodes nature (hot or cold) and on the other hand on two working parameters : the stabilization mode of the arc column and the nature of the used plasma gas (oxidant, neutral or reductant).

The last part deals high enthalpy plasma generators classified according to the used stabilization modes.
\end{abstract}

Introduction. - L'utilisation d'arcs électriques pour obtenir des gaz à très haute température date du début du siècle. Cependant l'activité dans ce domaine s'est développée considérablement depuis 1956, en raison des applications possibles dans le domaine de la simulation spatiale et de la propulsion [1]. Et ce n'est qu'au cours de ces dernières années que des applications industrielles dans les domaines des synthèses, du traitement des poudres, des traitements métallurgiques et des traitements de surface ont vu le jour.

Parmi les différentes sources de plasmas - telles que les décharges basse pression, les générateurs microondes, les générateurs hautes fréquences - les générateurs à plasma à arc soufflé occupent une position privilégiée. Ces générateurs peuvent en effet fonctionner à une pression égale ou supérieure à la pression atmosphérique. Les générateurs à faible 
enthalpie avec des dimensions réduites et d'un coût d'installation modéré permettent de transférer des puissances importantes - jusqu'à $3 \mathrm{MW}$ actuellement - à des masses de gaz allant jusqu'à $1 \mathrm{~kg} / \mathrm{s}$; tandis que ceux à forte enthalpie atteignent des puissances de l'ordre de $50 \mathrm{MW}$ et une enthalpie générée allant jusqu'à $10^{8} \mathrm{~kJ} / \mathrm{kg}$.

Dans cet article nous consacrons la première partie à l'étude des phénomènes rencontrés dans les générateurs à plasma en général. Nous présentons, après une définition rapide du plasma, les caractéristiques essentielles des arcs plasmas avec les phénomènes et les jets créés à la cathode et à l'anode. Les différentes méthodes de stabilisation de ces arcs (par la paroi, par transpiration, par injection tourbillonnaire d'un liquide ou d'un gaz ou par champ magnétique) sont étudiées ainsi que le comportement de l'arc dans un circuit. Les phénomènes propres aux électrodes dans les générateurs à plasma à courant alternatif sont brièvement indiqués et enfin l'usure des électrodes est discutée.

Dans une seconde partie nous présentons les principes de conception et de modélisation des générateurs à plasma et la forte influence des propriétés des gaz à haute température.

La troisième partie, après l'étude des caractéristiques principales des générateurs à plasma à faible enthalpie et de leurs utilisations, est consacrée à une revue des divers générateurs à plasma utilisés. La classification faite est liée d'une part à un paramètre de structure : nature des électrodes et d'autre part à deux paramètres de fonctionnement : mode de stabilisation de la colonne de l'arc et nature des gaz plasmagènes utilisés.

Enfin la dernière partie traite des générateurs à plasma à forte enthalpie selon les modes de stabilisation utilisés.

1. Définition et principes. - 1.1 LE Plasma. Le plasma est un milieu constitué d'un mélange de molécules d'atomes et d'ions le plus souvent dans des états excités, ainsi que d'électrons, l'ensemble étant électriquement neutre et les particules ayant dans la majorité des cas une grande énergie cinétique.

Si l'on excepte les lasers et les ondes de choc, la génération d'un plasma peut être schématiquement divisée en trois éléments fondamentaux : une source de puissance électrique, un couplage assurant le contact et une décharge ionisant l'atmosphère. D'une manière générale la source de puissance peut être soit un générateur électrique à courant continu ou alternatif, soit un générateur radiofréquence, soit un générateur microonde.

Pour les microondes (avec des fréquences de 200 à $3000 \mathrm{MHz}$ permettant au champ électrique de pénétrer dans le plasma [2]), le plasma est produit soit directement avec une cavité résonnante [2], soit par propagation d'une onde de surface [3].

Pour les hautes fréquences ( 1 à $20 \mathrm{MHz}$ en général) les ions n'atteignent plus les électrodes et leur accu- mulation augmente le champ électrique et donc le premier coefficient d'ionisation de Townsend ce qui facilite l'établissement de la décharge. Les électrodes peuvent alors être disposées hors de l'enceinte et le couplage est soit selfique [4], soit capacitif.

Dans cet article, nous ne traiterons ni des plasmas produits par microondes, ni de ceux obtenus par décharge radiofréquence mais des arcs pour lesquels le couplage est résistif, c'est-à-dire assuré par le gaz plasma en contact avec les électrodes aux bornes desquelles est appliquée la puissance. Une différence finie de potentiel aux électrodes favorise l'accumulation des charges au voisinage des électrodes et les fait accélérer. Ces charges, par collision, permettent le maintien de la décharge.

Les propriétés du plasma et de la décharge électrique dépendent de plusieurs paramètres notamment de la pression, du débit et de la nature du gaz utilisé, de la concentration en espèces chargées et de la température des électrons et du plasma et enfin des caractéristiques électromagnétiques telles que la tension, l'intensité du courant et le champ magnétique.

De très nombreux types de plasmas, aux propriétés physiques sensiblement différentes, suivant la pression et/ou l'intensité de la décharge, ont été décrits dans la littérature [5]. Leur état est essentiellement caractérisé par l'énergie des électrons $\left(k T_{\mathrm{e}}\right)$ et leur densité $\left(n_{\mathrm{e}}\right)$. Les plasmas de basse pression et/ou de faible intensité (par exemple ceux des décharges luminescentes ( $p \leqslant 100$ torrs) ou des décharges couronnes à la pression atmosphérique) sont entre autres caractérisés par un rapport entre la température des électrons $T_{\mathrm{e}}$ et celle du gaz $T_{\mathrm{g}}$ (particules lourdes) compris entre 10 et $10^{2}$. Cette absence d'équilibre permet d'obtenir un plasma dans lequel la température des gaz peut être voisine de l'ambiante alors que les électrons sont suffisamment énergétiques pour entraîner la rupture des liaisons moléculaires par exemple. Cette propriété rend ce type de décharge parfaitement adaptée aux réactions chimiques entre matériaux très sensibles aux effets de température (composés organiques par exemple). Quant aux arcs à haute pression (1 atmosphère et au-delà) et forte intensité, ils correspondent à une situation voisine de l'équilibre $\left(T_{\mathrm{e}}\right.$ et $\left.T_{\mathrm{g}}\right)$ sont presque identiques, et les températures élevées que l'on y rencontre $\left(T>4 \times 10^{3} \mathrm{~K}\right)$ rendent ce type de plasma adapté au traitement des matériaux non organiques : synthèses de réfractaires, projection et fusion, synthèses de petites molécules telles que l'acétylène, l'acide cyanhydrique ou les oxydes d'azote.

1.2 LES CARACTÉRISTIQUES ESSENTIELLES DES ARCS. 1.2.1 Généralités. - Une des différences essentielles entre les décharges luminescentes ou les décharges couronnes et les arcs électriques est la densité de courant qui est très forte dans la colonne de l'arc (de l'ordre de $100 \mathrm{~A} / \mathrm{cm}^{2}$ ) alors qu'elle est faible dans les décharges luminescentes ou couronnes $(1$ à $10 \mathrm{~mA} / \mathrm{cm}^{2}$ ). Dans les arcs cette densité de courant atteint $10^{6} \mathrm{~A} / \mathrm{cm}^{2}$ aux électrodes avec un flux de 
chaleur de $10^{6}$ à $10^{7} \mathrm{~W} / \mathrm{cm}^{2}$, ce qui implique, afin d'en limiter l'érosion, une protection efficace des électrodes permettant l'évacuation des calories. De même si la chute cathodique dans les arcs est d'une dizaine de volts, du fait de la très forte émission d'électrons (dépassant $10^{16}$ électrons $/ \mathrm{cm}^{3}$ ), elle atteint $100 \mathrm{~V}$ dans les décharges luminescentes.

La distribution du potentiel dans l'arc (Fig. 1) présente quant à elle quelques particularités. Le changement de potentiel est particulièrement important au niveau des électrodes, zones qui correspondent aux chutes anodique et cathodique. L'influence des électrodes intervient tant au niveau des zones anodiques et cathodiques que dans la colonne de l'arc qui peut être modifiée par les jets cathodiques et/ou anodiques.
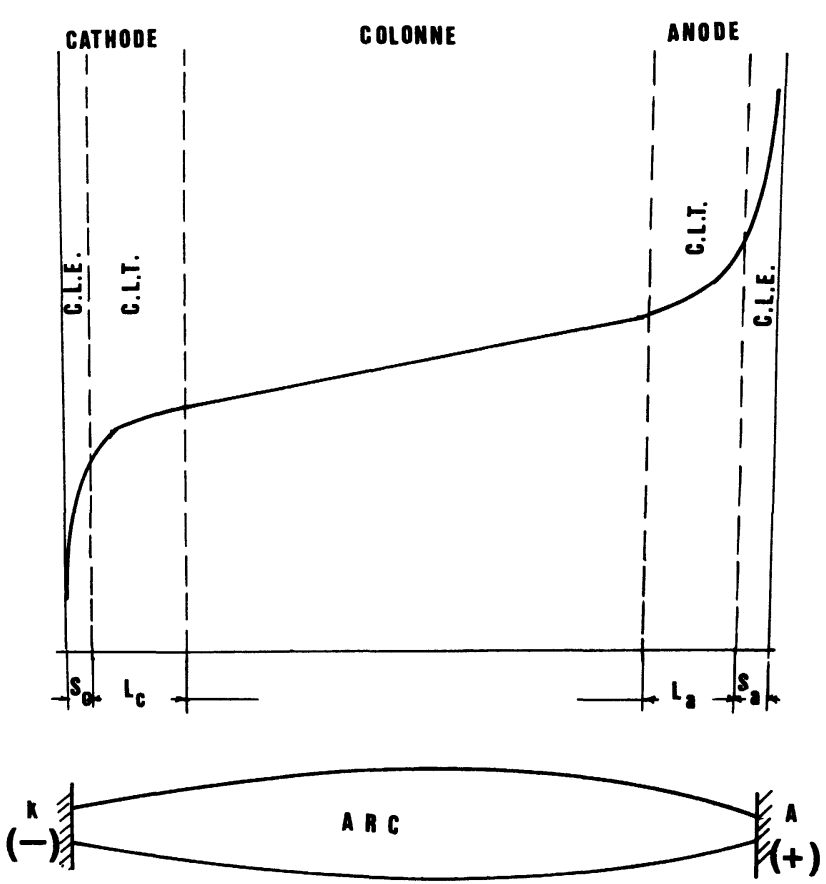

Fig. 1. - Distribution du potentiel dans l'arc.

[Potential distribution along an arc.]

Les zones anodiques et cathodiques sont ellesmêmes subdivisées en deux parties :

a) Les gaines électriques anodique $S_{\mathrm{a}}$ et cathodique $S_{\mathrm{c}}$ forment la frontière entre l'électrode solide ou liquide et le gaz plasma; elles assurent ainsi la transition électrique. Leur épaisseur est de l'ordre de la longueur de Debye $\lambda_{\mathrm{D}}$, elle est plus faible que le libre parcours moyen des électrons et atteint $10^{-4} \mathrm{~mm}$. Dans ces zones une déviation de la quasi-neutralité électrique se produit due à la charge spatiale induite par les champs électriques. Ces régions doivent être considérées comme formant une couche limite électrique (C.L.E.).

b) Les courbes de diffusion anodique $L_{\mathrm{a}}$ et cathodique $L_{\mathrm{c}}$ sont caractérisées par des gradients axiaux de température, de densité de particules et de rayon- nement très forts, gradients induits par la proximité de la paroi, ainsi que par des densités de courant plus élevées que dans la colonne de l'arc. Ces zones sont considérées comme la couche limite thermique (C.L.T.) à la paroi des électrodes. Leur épaisseur varie entre 0,1 et $1 \mathrm{~mm}$ selon la densité de courant dans la zone d'attache. Elles assurent la transition thermique entre la température élevée de la colonne de plasma et celle de la paroi ( $\sim 1000 \mathrm{~K})$. Le gaz plasma dans ces couches est électriquement neutre.

La compréhension, l'analyse et la modélisation de ces zones de transition électrique et thermique entre le plasma et la paroi présentent de sérieuses difficultés dues à la complexité des phénomènes se passant dans un espace très limité [6].

Ajoutons enfin que les effets gazodynamiques de ces zones aux électrodes créent les phénomènes de jets anodiques et cathodiques que nous expliquerons par la suite.

c) Enfin la colonne de l'arc forme la zone d'écoulement effectif du plasma, dans laquelle les gradients axiaux des différentes propriétés du plasma sont relativement faibles.

1.2.2 La cathode. - Définissons maintenant le rôle des électrodes d'un générateur de plasma à arc.

La cathode doit assurer une émission d'électrons et constituer un pôle d'attache de l'arc. Cet attachement peut se présenter sous deux formes :

a) Attachement sur une ou plusieurs taches cathodiques qui se déplacent lentement. - Dans ce mode d'attachement l'arc présente une constriction sévère près de la cathode qui fait apparaitre la zone de transition comme une tache avec des densités de courant liées à la nature du gaz et de l'ordre de $10^{2}$ à $10^{3} \mathrm{~A} / \mathrm{cm}^{2}$ pour les oxydes réfractaires tels que le $\mathrm{ZrO}_{2}$ [7], et de $10^{3}$ à $10^{4} \mathrm{~A} / \mathrm{cm}^{2}$ pour les métaux réfractaires tels que le tungstène thorié ou le zirconium. L'émission thermoionique des électrons est le mécanisme prédominant, elle se produit du fait d'une température de surface de la cathode relativement élevée $(>3500 \mathrm{~K})$. La forme de ces cathodes chaudes est déterminée par les intensités du courant à supporter [8]. Des cathodes en forme de tige à embout arrondi ou pointu de quelques millimètres à $15 \mathrm{~mm}$ de diamètre sont souvent utilisables pour des intensités ne dépassant pas $1000 \mathrm{~A}$, les pastilles embouties ou brasées dans un matériau bon conducteur tel que le cuivre sont utilisables pour des intensités pouvant atteindre $5000 \mathrm{~A}$, tandis que des cathodes creuses permettent de dépasser cette valeur en distribuant l'émission des électrons sur une surface relativement plus importante. L'érosion de ces cathodes est souvent rapide, elle se fait par sublimation ou évaporation, elle peut varier entre $10^{-8}$ à $10^{-10} \mathrm{~g} / \mathrm{C}$ [9]. Les problèmes d'érosion sont importants du fait des flux de chaleur spécifique élevés transférés à la cathode. Ils s'accentuent avec la constriction qui augmente d'ailleurs avec la pression. La limite maximale de pression pour un bon fonctionne- 
ment de ces cathodes chaudes ne doit donc pas dépasser $10^{3} \mathrm{~Pa}$. La majorité des cathodes est refroidie, soit par un emmanchement dans un support réfrigéré, soit par un gaz protégeant la cathode et confinant en même temps l'arc dans une position fixe, soit en utilisant les deux systèmes simultanément.

Ce mode d'émission thermo-ionique de la cathode peut être décrit par la loi de Richardson-Dushman [10] :

$$
J=A T^{2} \mathrm{e}^{-\phi_{\mathrm{e}} e / k T}
$$

avec $J$ densité du courant

$T$ température de la cathode $(\mathrm{K})$

$$
A=\frac{2 \pi e m k^{2}}{h^{3}}
$$

et qui est pour la plupart des matériaux égal à $6 \times 10^{5} \mathrm{~A} \mathrm{~m}^{-2} \mathrm{~K}^{-2}$, $m$ et $e$ étant respectivement la masse et la charge des électrons, $k$ la constante de Boltzmann, $h$ la constante de Planck et $\phi_{\mathrm{e}}$ est le potentiel d'extraction du matériau de la cathode.

b) Attachement diffus en plusieurs petits points dispersés sur la surface de la cathode et se déplaçant rapidement et erratiquement. - La densité de courant dans cette forme d'attachement varie entre $10^{6}$ et $10^{8} \mathrm{~A} / \mathrm{cm}^{2}$ avec un flux de chaleur spécifique dépassant $10^{6} \mathrm{~W} / \mathrm{cm}^{2}$ [11]. L'émission du champ électrique est non thermo-ionique, elle est le fait de températures ne dépassant pas $3000 \mathrm{~K}$. Les cathodes sont souvent faites en matériaux ayant une conductivité thermique élevée (tels que le cuivre ou ses alliages) permettant un refroidissement efficace, donc une diminution de l'érosion qui prend la forme d'une ébullition suivie d'un arrachement. Ce refroidissement énergique indispensable dans ces cathodes froides est cependant insuffisant si le pied de l'arc ne se déplace pas rapidement sous l'effet soit d'un écoulement gazeux tourbillonnaire, soit d'un champ magnétique extérieur, soit des deux à la fois. Le mouvement résultant de ce déplacement des points cathodiques assure un étalement du flux de chaleur sur une large section et réduit ainsi l'érosion de la cathode. Ces générateurs à cathodes froides ne sont pas sujets à une limitation de la pression de fonctionnement bien que l'érosion augmente avec l'accroissement de cette dernière.

Finalement nous avons résumé dans le tableau I les caractéristiques principales de ces deux types de cathodes.

Notons enfin que des effets secondaires dans cette région cathodique sont observés tels que des vapeurs ou des jets cathodiques (cf. $\S 1.2 .4$ ), effets secondaires qui exercent souvent une forte influence sur la région cathodique et sur l'arc tout entier.

1.2.3 L'anode. - L'anode, quant à elle, dissipe une grande partie de la puissance totale fournie à l'arc, ce qui exige donc une excellente protection thermique par un refroidissement efficace, ou par une récupération de la totalité de cette énergie dissipée en utilisant les charges à traiter (dans les fours par exemple) comme région anodique.

Cette zone anodique assurant la connection entre le plasma et le matériau à travers les couches limites thermiques et électriques joue les rôles suivants :

a) produire des ions pour maintenir le flux vers la cathode; ce flux est faible puisque la mobilité des ions est elle-même faible,

b) faire acquérir aux électrons une vitesse les dirigeant vers l'anode et permettant ainsi de les collecter.

Certains dispositifs utilisent des anodes consommables tels que le graphite ou le carbone qui s'érodent par sublimation, d'autres anodes sont protégées par un refroidissement énergique par eau ou par transpiration et sont réalisées avec des matériaux tels que le cuivre, certains de ses alliages, le molybdène, les aciers inoxydables dans certains cas ou le graphite poreux.

Les anodes ont en général des formes de symétrie de révolution telles que des tubes, des disques ou des

Tableau I. - Caractéristiques principales des cathodes.

\begin{tabular}{|c|c|c|c|c|c|c|c|c|c|c|c|}
\hline Cathode & $\begin{array}{c}\text { Attache- } \\
\text { ment }\end{array}$ & Emission & $\begin{array}{c}\text { Densité } \\
\text { de } \\
\text { Courant }\end{array}$ & Matériaux & $\begin{array}{c}\text { Température } \\
\text { de } \\
\text { fonction- } \\
\text { nement }\end{array}$ & $\begin{array}{c}\text { Mou- } \\
\text { vement }\end{array}$ & Erosion & $\begin{array}{c}\text { Constric- } \\
\text { tion }\end{array}$ & Forme & $\begin{array}{c}\text { Intensité } \\
\text { maximale }\end{array}$ & $\begin{array}{c}\text { Pression } \\
\text { de } \\
\text { fonction- } \\
\text { nement }\end{array}$ \\
\hline \multirow{2}{*}{ chaude } & \multirow{2}{*}{ tache } & \multirow{2}{*}{$\begin{array}{l}\text { thermo- } \\
\text { ionique }\end{array}$} & $10^{2}-10^{3} \mathrm{~A} / \mathrm{cm}^{2}$ & $\begin{array}{c}\text { oxydes } \\
\text { réfractaires } \\
\left(\mathrm{ZrO}_{2}\right)\end{array}$ & \multirow{2}{*}{$>3500$} & \multirow{2}{*}{ lent } & \multirow{2}{*}{$\mid \begin{array}{c}\text { sublimation } \\
\text { ou } \\
\text { évaporation }\end{array}$} & \multirow{2}{*}{ sévère } & \multirow{2}{*}{$\begin{array}{l}\text { tige } \\
\text { pastilles } \\
\text { creuse }\end{array}$} & \multirow{2}{*}{$\begin{array}{r}1000 \mathrm{~A} \\
5000 \mathrm{~A} \\
>5000 \mathrm{~A}\end{array}$} & \multirow{2}{*}{$\approx$ ambiante } \\
\hline & & & $10^{3}-10^{4} \mathrm{~A} / \mathrm{cm}^{2}$ & $\begin{array}{l}\text { métaux } \\
\text { réfractaires } \\
\left(\mathrm{W}+\mathrm{ThO}_{2}\right)\end{array}$ & & & & & & & \\
\hline froide & diffus & $\begin{array}{l}\text { non } \\
\text { thermo- } \\
\text { ionique }\end{array}$ & $10^{6}-10^{8} \mathrm{~A} / \mathrm{cm}^{2}$ & $\begin{array}{l}\text { métaux } \\
\text { refroidis } \\
(\mathrm{Cu}, \text { alliages } \\
\mathrm{Cu} . . .)\end{array}$ & $<3500$ & rapide & $\begin{array}{l}\text { ébullition } \\
\text { suivie } \\
\text { d'arrache- } \\
\text { ment }\end{array}$ & simple & cylindres & - & $\begin{array}{l}\text { pas de } \\
\text { limitation }\end{array}$ \\
\hline
\end{tabular}

[Main characteristics of the cathodes.] 
tuyères. La densité de courant à l'anode varie entre $10^{2}$ et $10^{5} \mathrm{~A} / \mathrm{cm}$ [12] et le flux de chaleur maximum transmis peut atteindre des valeurs de $10^{5}$ à $10^{6} \mathrm{~W} / \mathrm{cm}^{2}$ pour des arcs à intensités élevées [13, 14].

Ce flux de chaleur local à l'anode est donné par le modèle conventionnel souvent basé sur une chute de courant anodique positive :

$$
Q_{\mathrm{a}}=Q_{\text {con }}+Q_{\mathrm{r}}+j_{\mathrm{e}}\left(\frac{5}{2} \frac{k T_{\mathrm{e}}}{e}+V_{\mathrm{a}}+\phi_{\mathrm{a}}\right)
$$

$Q_{\text {con }}$ est le transfert de chaleur par convection et conduction

$Q_{\text {r }} \quad$ est le transfert de chaleur par rayonnement et le dernier terme représente l'énergie transférée à l'anode due aux électrons avec :

$T$. la température des électrons entrant dans la zone de chute anodique,

$V_{\mathrm{a}}$ la chute anodique positive,

$\phi_{\mathrm{a}}$ le potentiel d'extraction électrique qui est fonction du matériau,

$j_{\mathrm{e}} \quad$ la densité de courant à la surface de l'anode.

Mais une récente corrélation améliorant la précédente et prenant en compte les points suivants a été donnée par [15] :

a) le plasma près de la paroi est hors d'équilibre, la température des électrons étant beaucoup plus élevée que celle des particules lourdes qui doivent atteindre, sur une distance très faible, la température de la surface anodique [16]. Dans la couche limite anodique c'est la température élevée des électrons qui maintient un plasma conducteur. Par exemple la figure 2 donne d'après [17] la distribution des températures des électrons et des particules lourdes dans un arc dans l'argon pour une densité de courant donnée en fonction de la distance à la surface de l'anode.

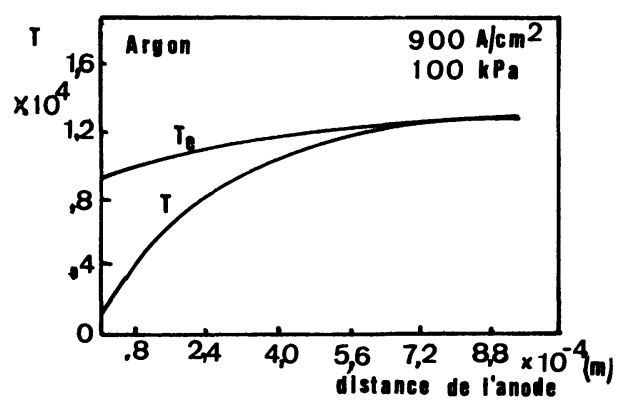

Fig. 2. - Evolution des températures des électrons $\left(T_{\mathrm{e}}\right)$ et des particules lourdes $(T)$ d'après [17].

[Heavy particle and electron temperature distribution in the anode boundary layer [17].]

L'épaisseur de la couche limite est donc déterminée par l'endroit où la température des particules lourdes atteint $90 \%$ de la température des électrons

$$
\left(T_{\mathrm{e}}-T=0,1 T_{\mathrm{e}}\right) \text {. }
$$

Elle a tendance à diminuer avec l'augmentation de la densité de courant électrique $« j$ ». Le tableau II en donne quelques exemples;

b) le champ électrique peut avoir des valeurs négatives sur presque toute l'étendue de la couche limite; dans ce cas la diffusion des électrons vers l'anode est retardée par la direction du vecteur du champ électrique. Cette situation résulte des gradients de température et de concentration extrêmement forts, elle induit des forces électro-motrices. L'effet de cette diminution brusque de la température et donc de la conductivité électrique du plasma à travers la couche limite anodique est contre-balancé par ces forces électro-motrices respectant ainsi la condition de la conservation du courant.

Par exemple, toujours dans un arc en atmosphère d'argon, la figure 3 montre une distribution de champ électrique dans la couche limite. Nous remarquons sa valeur positive loin de l'anode devenant légèrement négative dans la couche limite et fortement négative à la proximité de la surface de l'anode ;

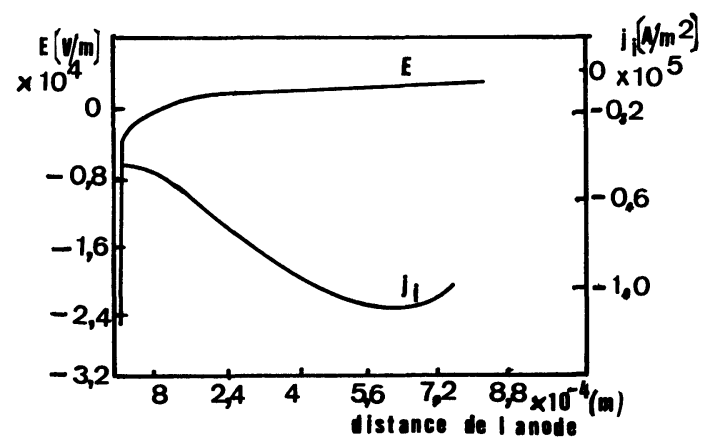

Fig. 3. - Evolution du champ électrique $(E)$ avec la distance de l'anode d'après [17].

[Electric field and positive current distribution in the anode boundary layer [17].]

c) la forte densité d'électrons à proximité immédiate de l'anode exige une chute de potentiel négative dans la couche électrique de gainage. La relation définissant cette chute est donnée par [18], elle est de la forme :

$$
\phi_{0}=-\frac{k T_{\mathrm{e}}}{e} \ln \left(\frac{e n_{\mathrm{e}} \bar{C}_{\mathrm{e}}}{4 J}\right)
$$

avec $n_{\mathrm{e}}, T_{\mathrm{e}}, \bar{C}_{\mathrm{e}}$ respectivement densité, température et vitesse moyenne des électrons, $J$ est la densité du courant.

Le tableau II en donne quelques exemples.

En tenant compte des trois remarques précédentes, le flux d'énergie total dissipé dans l'anode est alors donné par la relation suivante :

$$
\begin{aligned}
Q_{\mathrm{a}}=Q_{\text {con }}+Q_{\mathrm{r}}-k_{\mathrm{e}} \frac{\mathrm{d} T_{\mathrm{e}}}{\mathrm{d} x} & -k_{\mathrm{a}} \frac{\mathrm{d} T}{\mathrm{~d} x}+ \\
& +J\left(3,203 \frac{k T_{\mathrm{e}}}{e}+\phi_{\mathrm{a}}\right)
\end{aligned}
$$


le transfert de chaleur par convection $Q_{\text {con }}$ et par rayonnement $Q_{\mathrm{r}}$ ainsi que le terme de la conduction de chaleur des électrons sont généralement négligeables, sauf en ce qui concerne le terme de convection lorsque l'anode est située dans une zone d'écoulement fortement turbulente. Le quatrième terme représente, quant à lui, la conduction due aux particules lourdes.

Tableau II. - Valeurs de l'épaisseur de la couche limite anodique " $L$ " et de la chute de potentiel anodique $\phi_{0}$ en fonction de la densité de courant $J$, pour un plasma d'argon d'après [15]

[The values of the anode boundary layer thickness $L$ and the anodic potential drop $\phi_{0}$ as a function of the current density $J$, for an argon plasma [15].]

\begin{tabular}{ccc}
\multicolumn{3}{c}{$\begin{array}{c}\text { Epaisseur } « L » \\
(\mathrm{~mm})\end{array}$} \\
$J(\mathrm{~A} / \mathrm{cm})$ & couche limite & $\phi_{0}(\mathrm{~V})$ \\
- & - & $-2,2$ \\
600 & 0,53 & $-2,2$ \\
1000 & 0,32 & $-1,9$ \\
3000 & 0,12 & $-2,7$
\end{tabular}

La densité d’énergie $J$ est donnée dans ce cas par :

$$
j=\sigma\left(E+\frac{1}{e n_{\mathrm{e}}} \frac{\mathrm{d} p_{\mathrm{e}}}{\mathrm{d} x}\right)+\phi \frac{\mathrm{d} T_{\mathrm{e}}}{\mathrm{d} x}
$$

avec

$\sigma$ conductivité électrique,

$E$ champ électrique,

$p_{\mathrm{e}}$ pression partielle du gaz,

$\phi$ coefficient de diffusion thermique

elle contient donc, en plus du terme habituel de champ électrique, deux termes additifs.

La relation précédente (4) a l'avantage de prendre en considération l'effet du plasma hors d'équilibre près de la paroi, de la chute anodique et du champ électrique négatifs. Ceci se concrétise par la valeur de la constante dans le premier terme entre parenthèses qui devient 3,203 au lieu de 2,5, ainsi que par la nonapparition explicite du terme de chute anodique et donc la possibilité de prendre en compte les chutes de potentiel positives ou négatives.

1.2.4 Jets aux électrodes. - La formation des jets de plasma aux électrodes a été étudiée pour la première fois par Maecker [19]. Les jets cathodiques peuvent provenir en général de quatre sources différentes :

1) des jets induits électromagnétiquement,

2) de la vaporisation du matériau de la cathode et des impuretés de la surface cathodique,

3) de l'ablation du matériau de la cathode,

4) des réactions chimiques sur la surface de la cathode produisant des gaz.

Maecker a observé et expliqué les effets du pompage magnétique à la cathode. Un écoulement est en effet induit dans le cœur de la zone d'attache de l'électrode : les lignes de courant sont attirées vers la tache en raison de la contraction de l'arc, et une composante axiale de la force magnétique repousse le gaz plasma loin de l'électrode créant ainsi un jet cathodique soufflé. L'écoulement radial du gaz froid entrant refroidit les frontières de l'arc en créant ainsi une contraction plus prononcée et donc à nouveau un pompage plus fort.

L'effet de ce jet cathodique pour des intensités supérieures à $50 \mathrm{~A}$ permet de stabiliser l'arc car sa vitesse donnée par $V_{\max } \sim(j)^{1 / 2}$ devient alors notablement supérieure à celle des effets convectifs déplaçant erratiquement la tache cathodique.

Cet effet de jet cathodique est souvent prédominant, mais un effet similaire peut aussi exister à l'anode [20]. Le jet anodique induit par la constriction de l'arc dans la zone anodique peut contrôler entièrement les caractéristiques de la décharge dans cette région pour des intensités élevées et pour des distances interélectrodes suffisamment larges (hors de la zone d'action du jet cathodique).

1.3 Stabilisation de L'ARC Plasma. - La majorité des arcs électriques exigent pour un fonctionnement reproductible et non erratique un mécanisme de stabilisation créé soit extérieurement soit par l'arc lui-même. Ce mécanisme doit avoir pour effet de maintenir la colonne d'arc dans une position donnée stable et de forcer l'arc à un retour à sa position d'équilibre lors de toute déviation. Il faut remarquer que cette position stable n'est pas nécessairement une position stationnaire, l'arc pouvant par exemple tourner ou bouger le long des électrodes à une vitesse donnée. La stabilisation implique donc finalement que la colonne d'arc puisse uniquement se déplacer à l'intérieur d'une enveloppe de trajectoires bien définies, contrôlée par le mécanisme de stabilisation.

$\mathrm{Ce}$ mécanisme assure généralement par ailleurs une protection contre l'usure rapide des électrodes.

Notons enfin que la méthode de stabilisation de la colonne d'arc (il en existe cinq types actuellement que nous allons maintenant décrire) est liée directement au choix d'une conception adaptée d'un générateur à plasma.

1.3.1 Stabilisation par la paroi. - L'arc plasma est confiné dans un tube de section circulaire lui assurant ainsi une symétrie cylindrique. Toute déviation de la colonne d'arc vers la paroi est compensée par l'augmentation de la conduction de chaleur à la paroi ce qui a pour effet de réduire la température de la colonne et donc la conductivité électrique. L'arc est alors contraint de retourner à sa position d'équilibre.

Afin d'améliorer le refroidissement des parois de stabilisation pour des intensités élevées, on a été amené à utiliser des empilements d'étages métalliques (généralement en cuivre) refroidis par eau et isolés électriquement. Cet arrangement est connu sous le nom d'arc à cascade stabilisé par la paroi. Le schéma de la figure 4 montre le principe de cette configuration. 


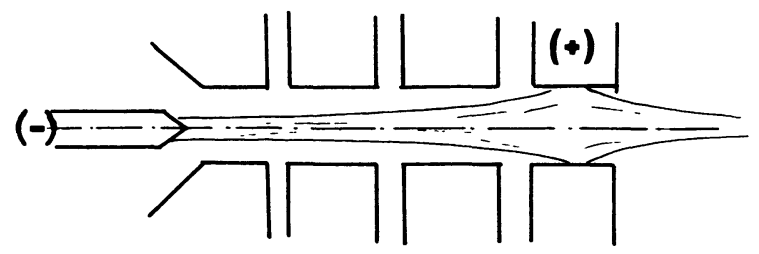

Fig. 4. - Principe de la stabilisation par la paroi segmentée.

[Schematic of a segmented wall stabilization.]

De plus ceci permet d'augmenter notablement la longueur de l'arc. En effet un simple tube métallique séparant l'anode de la cathode conduirait à l'amorçage de deux arcs, un de la cathode au tube, l'autre de l'anode au tube. Avec un dispositif segmenté, si $E$ est le champ électrique et $d$ l'épaisseur d'un segment, on doit avoir, pour éviter ce phénomène :

$$
\int_{0}^{d} E \mathrm{~d} x<V_{\mathrm{c}}+V_{\mathrm{a}}
$$

$V_{\mathrm{c}}$ et $V_{\mathrm{a}}$ étant respectivement les chutes anodiques et cathodiques.

Il est essentiel pour obtenir un effet de paroi stabilisant que le diamètre du constricteur soit plus petit que celui de l'arc libre fonctionnant dans les mêmes conditions. Si le diamètre du tube est très grand, l'effet stabilisant dû à la conduction de la chaleur est perdu et simultanément les effets de la convection libre créent des perturbations sérieuses de la colonne de l'arc. Il faut noter que dans de telles configurations l'enthalpie et la température maximales possible sont limitées par la valeur du flux de chaleur maximum admissible à la paroi confinante. Un refroidissement énergétique permet d'atteindre des flux de l'ordre de $10^{5} \mathrm{~kW} / \mathrm{m}^{2}$.

1.3.2 Stabilisation par transpiration. - Un refroidissement des parois par transpiration de gaz remplace le refroidissement par eau. Les flux de chaleur convectif/conductif en provenance de l'arc et se déplaçant vers les parois rencontrent un écoulement de gaz froid orienté radialement. Le gradient de température à la paroi est ainsi réduit. Ce mécanisme est très efficace tant que le transfert du plasma chaud vers la paroi d'un constricteur poreux est essentiellement dû à la conduction et la convection, mais ce mode de refroidissement perd son efficacité si le rayonnement devient prédominant, les instabilités obtenues créent une forte variation de la température le long de la paroi du canal. L'obtention d'une distribution plus uniforme de cette température peut être assurée par un montage segmenté assurant un débit régulier de gaz à travers la paroi. La figure 5 présente schématiquement le principe $[21,22,23]$. D'une manière générale ce type d'arc permet d'obtenir des puissances par unité de longueur d'arc très supérieures à celles obtenues avec la configuration précédente et donc des températures de gaz encore plus élevées, pouvant atteindre dans le cas de l'hydrogène et si la tenue des électrodes le permet une température de $60000 \mathrm{~K}$.

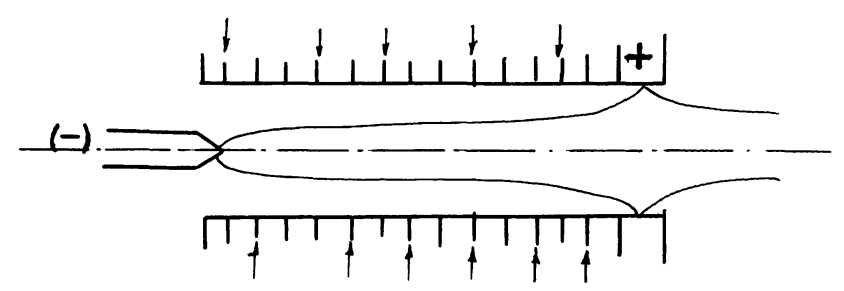

Fig. 5. - Principe de la stabilisation par transpiration. [Schematic of the stabilization by transpiration walls.]

1.3.3 Stabilisation par injection tourbillonnaire. a) d'un liquide : dans le cas de l'injection tourbillonnaire l'arc est confiné au centre du tube dans lequel un vortex du liquide est maintenu plaqué le long de la paroi par les forces centrifuges y assurant ainsi la protection thermique (Fig. 6). L'arc plasma est généré par les vapeurs produites dans le coeur du vortex [24]. La puissance dissipée par unité de volume et donc la température peuvent atteindre des valeurs très élevées étant donné le très bon refroidissement du contour de l'arc. Des températures de l'ordre de $50000 \mathrm{~K}$ ont été mesurées par [25] pour un arc ayant une intensité de $1450 \mathrm{~A}$ et confiné dans un diamètre $<2,3 \mathrm{~mm}$.

b) d'un gaz : le fluide de fonctionnement est le gaz ou les mélanges gazeux injectés tangentiellement et créant le vortex recherché (Fig. 7). Les électrodes sont refroidies par une circulation d'eau. Des jets cathodiques et anodiques sont créés provoquant souvent une érosion des électrodes. Ce vortex assure par les forces de frottement importantes entre la paroi et le fluide l'élimination des effets de convection [26-30].



Fig. 6. - Principe de la stabilisation par injection tourbillonnaire de liquide.

[Schematic of the liquid-vortex stabilized arc.]

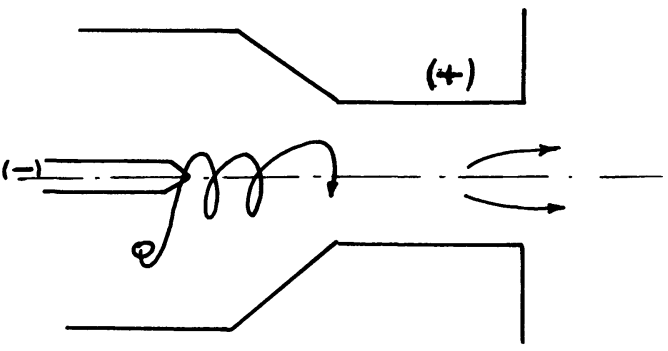

Fig. 7. - Principe de la stabilisation par injection tourbillonnaire du gaz.

[Schematic of the gas-vortex stabilized arc.] 
1.3.4 Stabilisation par champ magnétique. - L'arc plasma, milieu électriquement conducteur, peut interagir avec son propre champ magnétique si son intensité est $>10000 \mathrm{~A}$, mais aussi avec des champs magnétiques extérieurs qui lui sont appliqués (Fig. 8).

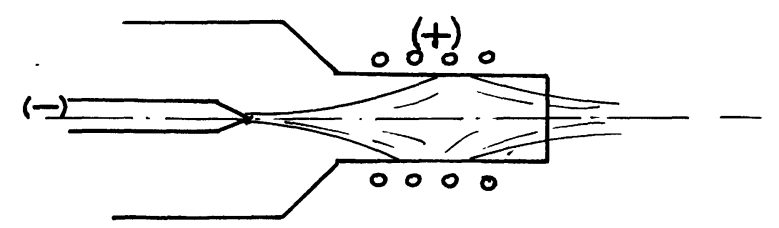

Fig. 8. - Principe de la stabilisation par champ magnétique extérieur.

[Schematic of the external magnetic field stabilization.]

Par exemple les bobines du champ magnétique entourant les électrodes créent un champ magnétique ayant une composante perpendiculaire à la direction du courant. Les forces $\mathbf{j} \times \mathbf{B}$ dans cette zone entraînent l'arc dans la direction azimuthale et une vitesse constante de cet arc magnétisé est établie par un équilibre entre les forces motrices magnétiques et les forces de traînée agissant sur la colonne de l'arc et qui sont dues au mouvement relatif du gaz.

Cette vitesse peut être calculée d'après l'équation donnée par [31] :

$$
U=4,6 B^{0,6} I^{0,4} d^{-0,4} \rho^{-0,5}
$$

avec

$B$ valeur du champ magnétique,

$I$ intensité du courant,

$d$ distance entre les électrodes,

$\rho$ densité à la pression atmosphérique.

Ce dispositif, dont la consommation énergétique ne dépasse jamais $10 \%$ de l'énergie totale fournie à l'arc, offre quatre avantages qui rendent les générateurs à plasma correspondants très attractifs pour leur utilisation dans les procédés chimiques :

1) la rotation de l'arc distribue la charge d'énergie à l'anode et réduit ainsi son érosion,

2) le niveau d'enthalpie des gaz chauds peut être facilement contrôlé en variant l'intensité de l'arc et la vitesse de rotation,

3) des volumes relativement importants de gaz peuvent être chauffés à des températures à peu près uniformes,

4) la rotation de l'arc augmente la chute du potentiel et permet de dissiper une puissance plus importante pour une même intensité.

1.4 L'ARC DANS UN CIRCUIT. - Le comportement de l'arc dans le circuit électrique d'alimentation est instable sans une résistance $R$ de stabilisation ajoutée en série dans le circuit. Nous avons alors :

$$
U_{\mathrm{a}}+R I=V_{\mathrm{s}}
$$

où

$U_{\mathrm{a}}$ tension aux bornes de l'arc,

$V$, tension délivrée par la source de tension.

En représentant la variation de $U_{\mathrm{a}}$ et de $R I$ en fonction de l'intensité $I$ du courant sur la figure 9, on remarque que le fait d'ajouter une résistance en série a pour effet de relever la partie décroissante de la caractéristique. Lorsque la tension de source $V_{\mathrm{s}}$ est indépendante de $I$ dans la zone de fonctionnement, le point de fonctionnement se situe alors à l'intersection de la droite $V_{\mathrm{s}}=$ Cte et de la nouvelle caractéristique. Seul le point correspondant à l'intensité la plus élevée conduit à un fonctionnement stable. En effet seule la partie croissante de la caractéristique est susceptible de conduire à des arcs stables, la limite supérieure étant atteinte lorsque la puissance dissipée conduit à des densités de courant trop importantes pour les électrodes. On peut ainsi définir la zone de fonctionnement stable (par exemple la partie droite de la figure 9). Cette résistance de stabilisation $R$ doit dépasser $30 \%$ de la valeur de la résistance de l'arc [32].

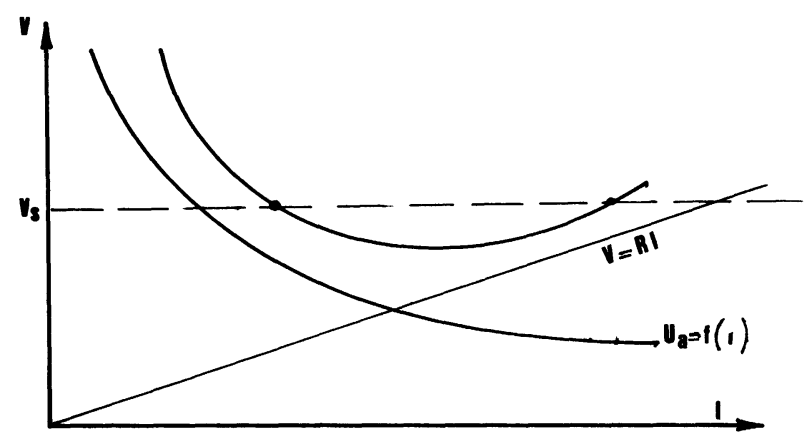

Fig. 9. - Relèvement de la caractéristique descendante de l'arc par adjonction d'une résistance $R$ en série dans le circuit électrique.

[The influence on the arc characteristics of the resistance $R$ added in series to the electric circuit.]

1.5 Le Plasma en courant alternatif. - Le changement de polarité dans les arcs plasmas en courant alternatif crée fréquemment des phénomènes complexes aux électrodes qui compliquent la compréhension des caractéristiques de l'arc. Tout d'abord ils ne peuvent fonctionner avec des électrodes chaudes car une cathode en matériau réfractaire avec émission thermo-ionique ne peut pas devenir facilement anode, le temps de refroidissement étant très supérieur à la période du courant.

C'est pourquoi les électrodes sont souvent en cuivre refroidi, et la configuration est celle d'électrodes tubulaires avec stabilisation combinée magnétique et tourbillonnaire [33].

Par ailleurs afin d'éviter l'extinction de l'arc lors de changement de phase, une tension élevée (qui peut atteindre 40 à $60 \mathrm{kV}$ ) soit par pulses, soit en haute fréquence est généralement superposée à l'arc assurant ainsi le réallumage [34]. Cependant pour un maintien 
correct de l'arc on est souvent amené à avoir une puissance $\mathrm{HF}$ qui peut atteindre $10 \%$ de la puissance de l'arc. Un tel dispositif est utilisé par [35] qui décrit un générateur de $100 \mathrm{~kW}$ fonctionnant à $50 \mathrm{~Hz}$ avec des électrodes annulaires en cuivre et un champ magnétique de $0,5 \mathrm{~Wb} / \mathrm{m}^{2}$ faisant tourner l'arc à raison de $10^{5} \mathrm{t} / \mathrm{s}$. Il est également possible de s'arranger pour que la distance séparant les deux électrodes tubulaires et la tension à vide d'alimentation soient telles que le réamorçage se fasse naturellement par claquage comme dans le dispositif Westinghouse. Il existe aussi des arcs triphasés qui éclatent entre trois électrodes refroidies disposées à $120^{\circ}$. Le démarrage peut en être assuré en créant une zone conductrice avec un jet de plasma pilote produit avec un générateur continu $[36,37]$ (la puissance de ce dernier étant très faible devant la puissance dissipée dans l'arc triphasé).

Citons également un arc à trois électrodes toriques fonctionnant en triphasé [38].

Il faut noter alors que compte tenu des dispositifs annexes nécessaires au fonctionnement de tels arcs leur coût, source comprise, est pratiquement identique

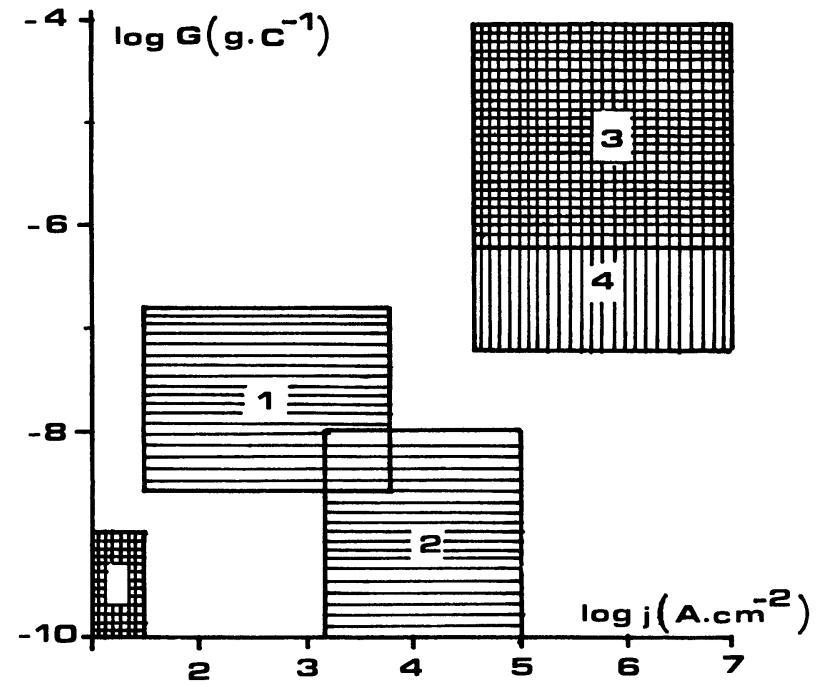

Fig. 10. - L'érosion spécifique fonction de la densité de courant pour des électrodes fonctionnant sous des atmosphères réductrices ou neutres et sous différents régimes, d'après [40].

Zone 1 : Cathodes en oxydes réfractaires et en régime thermoionique.

Zone 2 : Cathodes en métaux réfractaires et en régime thermoionique.

Zone 3 : Cathodes en régime diffus.

Zone 4 : Anodes.

Zone 5 : Cathodes et anodes pour une faible densité de courant et en régime diffus.

[The specific erosion and the current density values for electrodes working in reducing or neutral atmospheres and under different conditions (after [40]).

Zone 1 : Refractory oxides cathodes and thermoionic regime. Zone 2: Refractory metals cathodes and thermoionic regime. Zone 3 : Cathodes in a diffused regime.

Zone 4 : Anodes.

Zone 5 : Cathodes and anodes for a low current density and a diffused regime.] à celui des arcs continus, qui de plus conduisent généralement à des usures d'électrodes plus faibles.

1.6 L'USURE Des ÉLECTRODES. - Pour les opérations industrielles, la durée de vie des électrodes est un élément primordial. Une durée moyenne de $200 \mathrm{~h}$ est en général atteinte sans difficulté dans les atmosphères non oxydantes mais elle n'excède pas $100 \mathrm{~h}$ quand les cathodes fonctionnent avec des gaz réactifs (oxygène, air, chlore). Par ailleurs l'utilisation continue d'électrodes consommables, comme celles utilisées pour le crackage des produits pétroliers, peut atteindre plusieurs centaines d'heures d'opération sans interruption [39].

La figure 10 donne d'après [40] l'érosion en fonction de la densité de courant de l'arc pour des cathodes et anodes ayant diverses formes d'attachement (cf. $\$ 1.2 .2$ et tabl. I).

La protection contre l'usure peut être améliorée notamment par l'une des méthodes de stabilisation citées précédemment (cf. $\S 1.3$ ). Nous donnons en exemple la figure 11 d'après [41] représentant l'influence de la vitesse de rotation de l'arc sur l'usure des électrodes pour différentes intensités du courant et divers matériaux. Il est à noter que l'usure n'est pas une simple fonction de la vitesse de rotation de l'arc et que l'augmentation de cette dernière n'améliore pas forcément la durée de vie de l'électrode [42], ce qui implique donc par exemple l'adaptation du champ magnétique de stabilisation aux débits de gaz et à la puissance de l'arc.

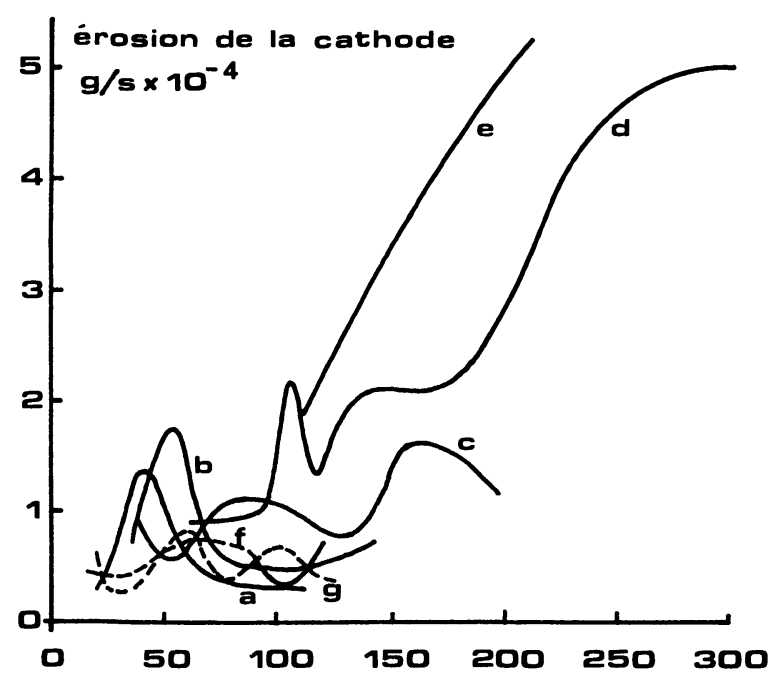

Fig. 11. - Erosion des électrodes avec la vitesse de rotation de l'arc pour différentes intensités du courant et divers matériaux [41]. a) $45 \mathrm{~A}, 420 \mathrm{~s}, \mathrm{Cu}$

b) $100 \mathrm{~A}, 420 \mathrm{~s}, \mathrm{Cu}$

c) $220 \mathrm{~A}, 120 \mathrm{~s}, \mathrm{Cu}$

d) $405 \mathrm{~A}, 100 \mathrm{~s}, \mathrm{Cu}$

e) $800 \mathrm{~A}, 30 \mathrm{~s}, \mathrm{Cu}$

f) $45 \mathrm{~A}, 120 \mathrm{~s}, \mathrm{~W} / \mathrm{Ag}$

g) $45 \mathrm{~A}, 120 \mathrm{~s}, \mathrm{~W} / \mathrm{Cu}$.

[Electrode erosion with the arc speed rotation for several current intensities and materials [41].] 
2. Principes de conception des générateurs à plasma. - 2.1 LES PROPRIÉTÉS DES GAZ À HAUTE TEMPÉRATURE. - La conception des générateurs à plasma est fortement liée à l'usage auquel est destiné le plasma produit et donc aux propriétés de ces gaz chauds ou de leurs mélanges. Dans le cas des réactions hétérogènes, qui sont d'abord fonction des échanges thermiques, il est essentiel de connaître la conductibilité thermique du plasma utilisé. La figure 12 présente l'évolution de la conductibilité thermique en fonction de la température pour des mélanges gazeux d'après [43]. Nous pouvons remarquer que l'ajout d'hydrogène à des plasmas d'argon ou d'azote permet d'augmenter sensiblement la valeur de cette conductibilité jusqu'à des températures de l'ordre de $12000 \mathrm{~K}$, et par conséquent d'augmenter les possibilités d'échange thermique entre le plasma et les corps que l'on veut y faire réagir. Ces échanges d'ailleurs dépendent de la conductivité thermique intégrée

$$
\bar{k}=\frac{1}{T_{\mathrm{p}}-T_{\mathrm{s}}} \int_{T_{\mathrm{s}}}^{T_{\mathrm{p}}} k \mathrm{~d} t
$$

représentée sur la figure 13 pour l'hydrogène, l'azote et l'argon d'après [44]. Nous remarquons la présence d'une température minimale au-delà de laquelle le plasma présente des valeurs de conductibilité élevées. Cette température est de l'ordre de $7000 \mathrm{~K}$ pour $\mathrm{N}_{2}$, de $4000 \mathrm{~K}$ pour $\mathrm{H}_{2}$ et se déplace vers les plus basses températures avec l'adjonction de gaz plus facilement dissociables comme $\mathrm{CH}_{4}$ par exemple.

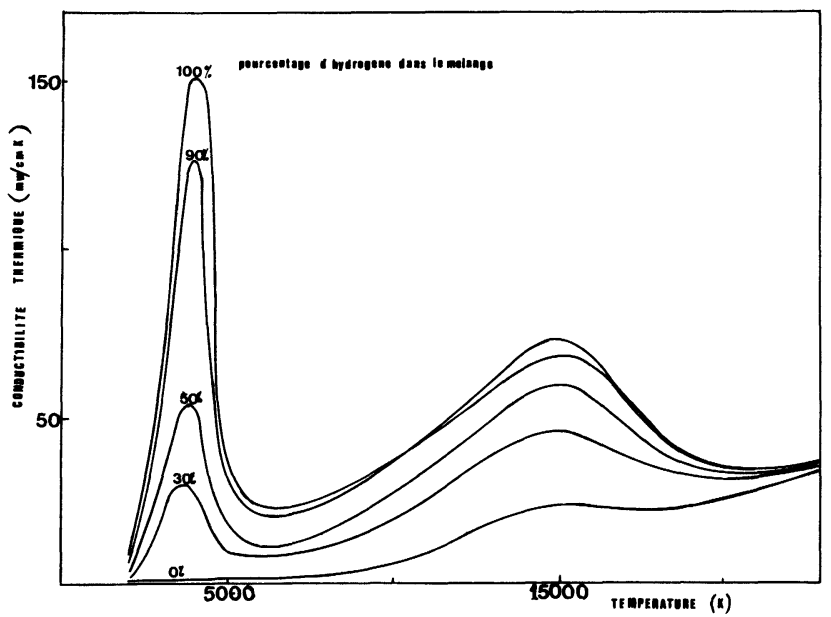

Fig. 12. - La conductivité thermique totale pour un mélange d'hydrogène et d'argon d'après [43].

[Thermal conductivity for hydrogen, argon mixture [43].]

Par ailleurs la valeur de la viscosité d'un plasma d'arc est importante (10 fois celle du gaz froid environ), ce qui pose souvent des problèmes pour la pénétration des particules solides ou des réactifs gazeux dans le plasma. La figure 14 donne son évolution avec la température pour les gaz plasma les plus utilisés.

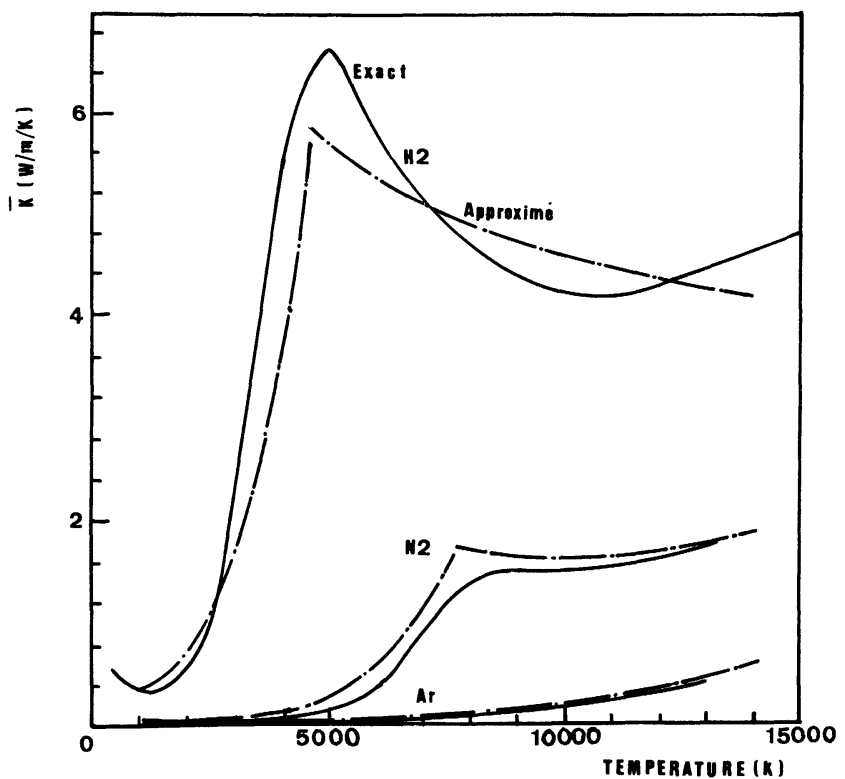

Fig. 13. - Evolution de la conductibilité thermique moyenne d'après [44].

Exact

$\bar{k}=\frac{1}{T-300} \int_{300}^{T} k(t) \mathrm{d} t$.

Approximé - - - -

$\bar{k}=\frac{1}{T-300} 10^{A T^{B}}+C$.

[Mean thermal conductivity [44].]

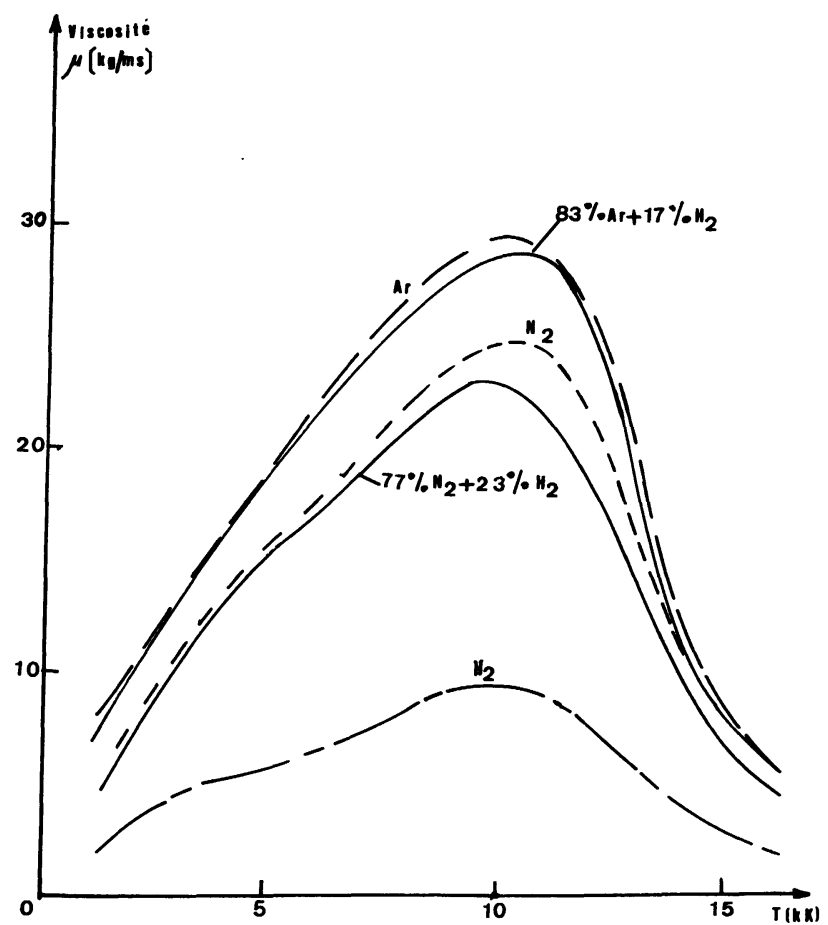

Fig. 14. - Viscosité des mélanges plasmagènes.

[Viscosity of plasma mixtures.] 
Enfin la conductivité électrique du plasma permettant de définir les propriétés électriques des gaz ionisés est donnée sur la figure 15 , sa valeur augmente sensiblement avec la température ce qui bien évidemment rend les gaz chauds conducteurs électriquement, mais elle dépend peu de la nature du gaz aux températures élevées.

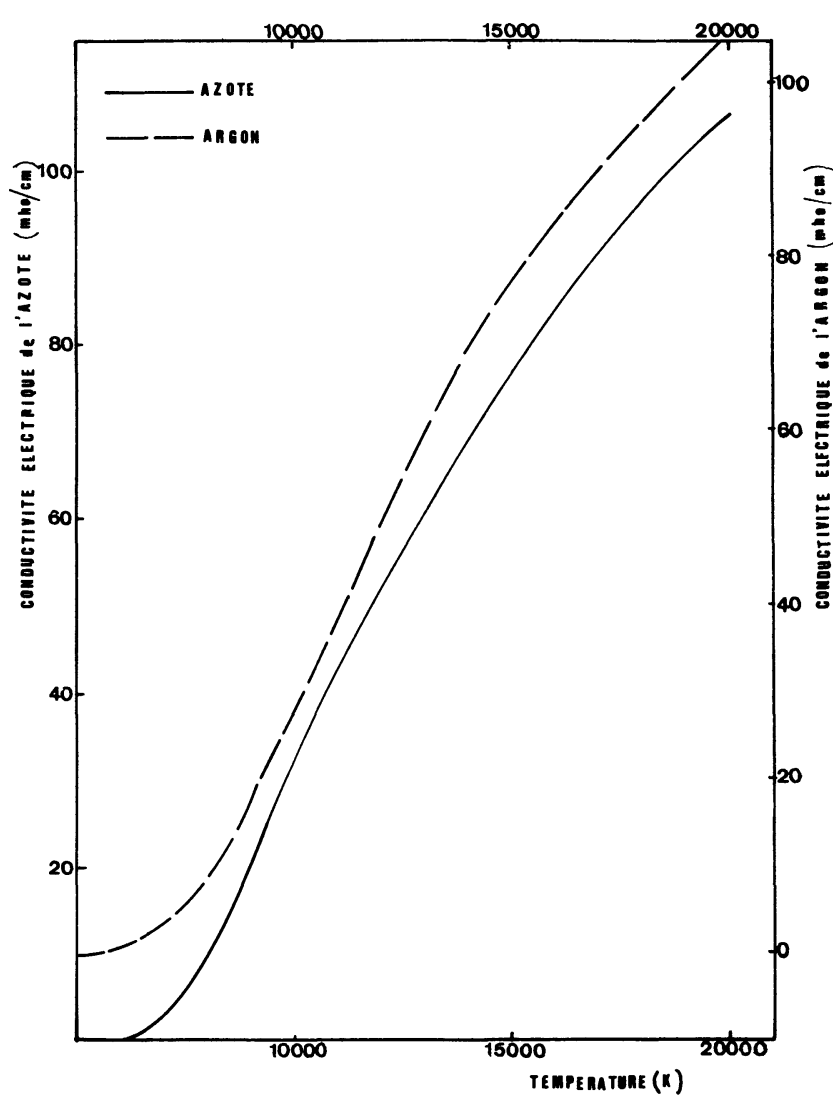

Fig. 15. - Evolution de la conductibilité électrique d'après [43]. [Electrical conductivity of a nitrogen and argon plasma [43].]

2.2 Modélisation Des géNÉRATEURS À Plasma. Comme nous l'avons vu précédemment $(\$ 1.2 .1)$ l'arc peut être divisé en trois parties : la région cathodique, la colonne plasma et la région anodique. La modélisation de la région cathodique est quasi impossible si l'on excepte l'approche qualitative donnée au (\$ 1.2.2) et l'évaluation de la densité de courant. Quant à la région anodique, l'approche faite par [17] présente un début de modélisation prenant en considération l'influence du plasma hors d'équilibre ( $\$ 1$ 1.2.3). Par ailleurs les phénomènes de fusion et d'ablation à l'anode et l'influence des vapeurs métalliques commencent à être pris en compte [45].

Enfin la modélisation de la colonne plasma est faite par la résolution des équations électro-magnétodynamique avec des conditions aux limites appropriées. Ceci permet de déterminer les caractéristiques de la colonne d'arc, de définir l'écculement plasmagène dans le réacteur et d'obtenir des relations semi-empiriques pouvant être utilisées pour la détermination des différentes valeurs géométriques nécessaire à la conception des réacteurs à gaz plasmagène. Ces équations comprennent [46] :

1) les équations de Navier-Stokes introduisant l'influence du mouvement du plasma selon les coordonnées axiales et radiales,

2) l'équation de continuité définissant la conservation de la masse,

3) l'équation du transfert d'énergie englobant l'influence du transfert par convection, par conduction, par rayonnement et par chauffage ohmique,

4) les équations électrodynamiques : équation de Maxwell...

5) les équations thermodynamiques,

6) les relations des propriétés thermophysiques avec la température.

A partir de ce système d'équations et en connaissant ou en supposant un profil de température et de vitesse en sortie de la région cathodique, nous pouvons déterminer :

- les isothermes de l'évolution de la température dans le réacteur étudié ainsi que la variation de celles-ci dans les directions axiale $\| x$ » et radiale $\| r$,

- les températures moyennes du gaz dans les différentes sections du canal,

- l'évolution des vitesses dans les directions axiale et radiale,

- les isovortex et les isocourants de l'écoulement,

- l'évolution du flux de chaleur à la paroi,

- l'évolution du nombre de Nusselt définissant ce transfert à la paroi,

- les valeurs de la longueur thermique développée,

- les caractéristiques électriques : intensités du champ électrique, etc...

Dans la pratique l'écoulement du gaz dans le générateur peut être schématisé sous deux formes : d'une part l'écoulement d'un gaz ou d'un mélange gazeux injecté froid s'ionisant et donc s'échauffant lors de son passage dans le champ électrique (entre l'électrodecathode et le tube anode) et d'autre part l'écoulement de ce gaz plasmagène en extinction dans la suite du canal au-delà de l'anode où il n'y a plus d'apport d'énergie (zone de champ libre) (Fig. 16).

Le gaz froid injecté près de la cathode traverse une première région, région interne dans laquelle le gaz

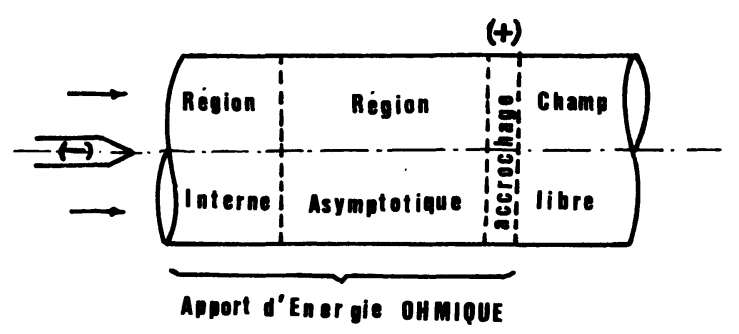

Fig. 16. - Schéma de principe des différentes zones de l'écoulement dans un réacteur plasma.

[Schematic of the flow regions in a plasma reactor.] 
chauffé électriquement et ionisé n'est pas encore en contact avec les parois refroidies.

La région asymptotique ou région pleinement développée, constitue la région suivante correspondant au développement et à la stabilisation de l'écoulement constricté par le contact avec les parois énergiquement refroidies.

Ces deux premières régions constituent les zones dans lesquelles on constate un apport d'énergie ohmique. Cette augmentation de l'énergie donc de la température du gaz s'arrête dans la zone d'accrochage anodique.

Dans cette zone le gaz plasmagène cède une partie de son énergie, et subit donc un gradient de température très élevée. La localisation de cette zone est l'une des difficultés essentielles. Pour le calcul pratiquement le seul moyen est de la définir précisément en imposant une isolation de l'anode par rapport au reste du canal.

L'écoulement devient enfin plus stable dans la région du champ libre. Le canal formant cette région est refroidi de façon homogène et on ne constate plus d'apport d'énergie ohmique dans cette région.

Il est à noter que ce modèle, tout en se plaçant dans une situation proche de la pratique, ne tient pas compte directement des jets cathodiques et anodiques présentés au (§1.2.4).

Par ailleurs la forme des profils de vitesse et de température supposés à l'entrée n'est importante que sur une faible longueur correspondant au début de la région interne [47].

Les quelques rares résolutions [48 à 52] faites de ce système introduisent un nombre important de simplifications et permettent l'obtention d'une solution numérique dans le cas d'un écoulement laminaire du gaz plasma. La figure 17 donne un exemple de l'évolution de la température qui augmente rapidement à l'entrée (dans la région interne) en atteignant un maximum; elle se stabilise ensuite le long de la région

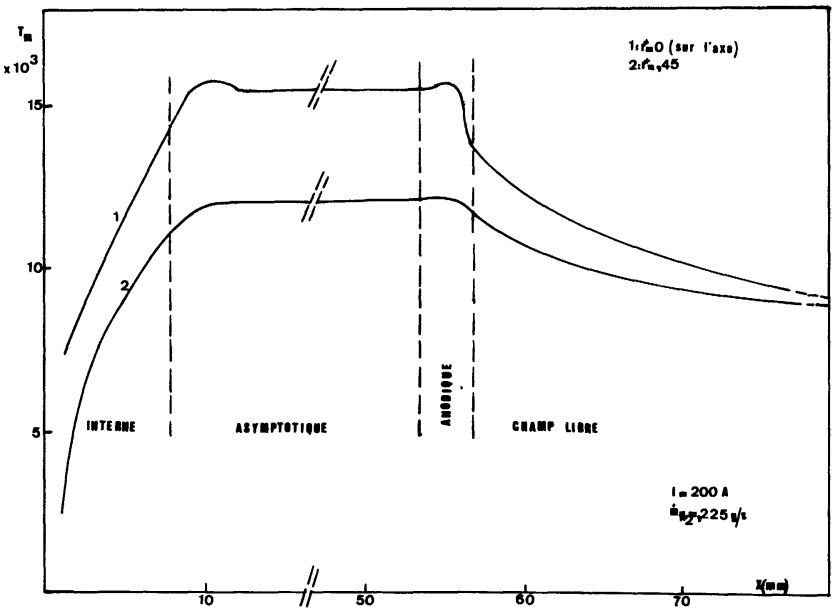

Fig. 17. - Profils axiaux de la température dans un canal d'après [46].

[Axial temperature profile in a circular duct [46].] asymptotique [53]. Quant au débit massique représenté sur la figure 18, il est concentré dans la couche relativement froide près de la paroi; ceci est dû à la faible densité massique du coeur de l'arc en raison de sa température élevée. Ce qui explique donc que dans une opération chimique le traitement total des gaz injectés se heurte à des difficultés importantes. La distribution massique s'égalise au fur et à mesure que l'on s'éloigne de l'entrée du canal donc avec le refroidissement du plasma.

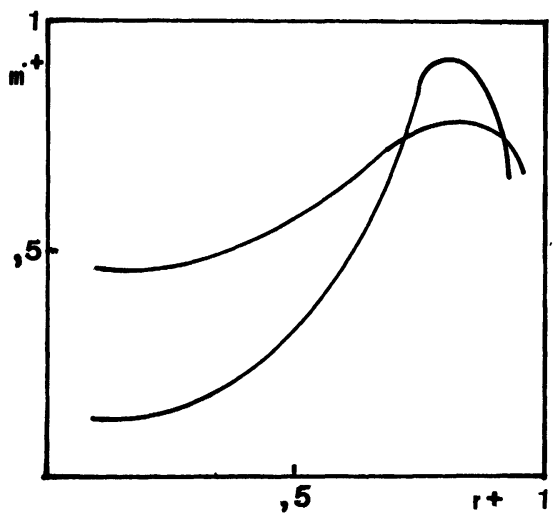

Fig. 18. - L'évolution radiale du débit massique du gaz dans différentes sections du canal d'après [46].

[Radial evolution of the mass flow rate in the different sections of the channel [46].]

Par ailleurs quelques relations semi-empiriques ont été déterminées pour différents gaz concernant le transfert de chaleur à la paroi d'un arc constricté, notamment l'évolution du flux de chaleur à la paroi et du nombre de Nusselt. Par exemple le flux de chaleur dans la région asymptotique est donné en fonction de l'intensité du courant et du diamètre pour l'hydrogène par :

$$
\varphi_{\mathrm{a}}=420 \frac{I}{D} \quad \text { d'après [46] } .
$$

Quant au nombre de Nusselt dans cette même région, il est déterminé en fonction du nombre de chauffage ohmique " $O H$ " par

$$
\begin{aligned}
N u_{\mathrm{a}} & =0,095 O H^{-1,018} & \text { pour } & \mathrm{H}_{2} \text { d'après [46] } \\
N u_{\mathrm{a}} & =0,135 O H^{-1,0} & \text { pour } & \text { Ar d'après [54] } \\
N u & =\frac{1,145}{\pi^{2}} O H^{-1,0} & \text { pour } & \mathrm{N}_{2} \text { d'après [55]. }
\end{aligned}
$$

Ces relations facilitent la modélisation de différents réacteurs à plasma et la détermination des variables géométriques, en prenant en compte le mieux possible les divers paramètres influents.

3. Générateurs à faible enthalpie. - 3.1 CARACTÉRISTIQUES PRINCIPALES ET UTILISATIONS. - Le rendement des générateurs à plasma à faible enthalpie 
présente une importance particulière dans les diverses utilisations. Ces générateurs fonctionnent en général à la pression atmosphérique et pour des durées longues. Leur puissance peut varier de quelques kilowatts à plusieurs mégawatts et leur densité d'énergie thermique atteint $10^{4} \mathrm{~kJ} / \mathrm{kg}$. Les débits de gaz injectés varient aussi de quelques litres par minute à quelques mètres cubes par minute. On peut noter que pour une même énergie fournie, et compte tenu des phénomènes de dissociation, la température obtenue est plus faible avec les gaz diatomiques qu'avec les gaz mono-atomiques, comme on peut le constater sur la figure 19 représentant, à la pression atmosphérique et d'après Baddour [56], l'enthalpie de différents gaz en fonction de la température.

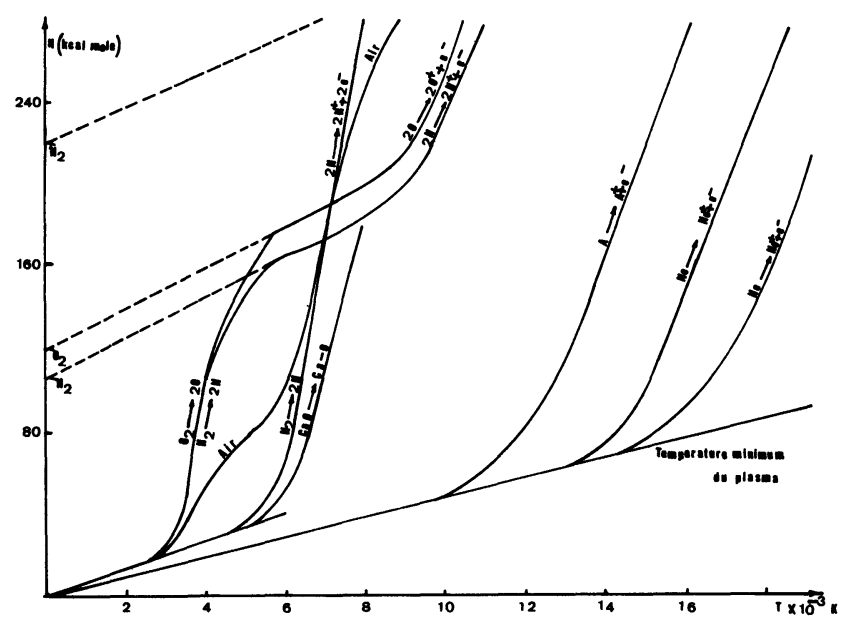

Fig. 19. - Evolution de l'enthalpie de divers gaz en fonction de la température d'après [56].

[Evolution of the gas enthalpy with the temperature after Baddour [56].]

Ces générateurs à plasma ont trouvé de multiples applications industrielles [57] notamment :

- la projection de revêtements protecteurs [58 et 59],

- la sphéroïdisation de céramiques (zircone, magnétite pour photocopie, ferrosilicium, etc...) [60 à 64],

- le découpage [65],

- la soudure [66],

- les synthèses de poudres ultra-fines (pigments de dioxyde de titane, poudre sphérique de silice à surface spécifique élevée, catalyseurs...) [67, 68],

- le crakage des produits pétroliers [69],

- comme source d'énergie thermique :

- dans les fours de refusion [70-75],

- pour la production d'aciers ou d'alliages spéciaux [76-83],

- pour la production de silicium ultra-pur, etc... $[84,85]$.

Nous allons maintenant présenter les divers générateurs à plasma utilisés en précisant leurs caractéristiques spécifiques. La classification que l'on peut en faire est liée d'une part à un paramètre de structure : nature des électrodes et d'autre part à deux paramètres de fonctionnement : mode de stabilisation de la colonne de larc et nature des fluides plasmagènes utilisés.

3.2 GÉNÉRATEURS À ÉLECTRODES chaudes. - Les électrodes chaudes réfractaires correspondent à l'existence d'une tache cathodique (cf. § 1.2.2.a). Pour les fortes intensités $(I>700 \mathrm{~A})$ avec l'utilisation de cathodes noyées dans du cuivre fortement refroidi (cf. $\S 1.2 .2 . a$ ) il est nécessaire que le pied d'arc soit centré sur le réfractaire par une injection tourbillonnaire au voisinage de la cathode.

Les matériaux les plus utilisés dans la fabrication des cathodes chaudes et dans le cas de l'atmosphère non oxydante (argon, hydrogène, azote, hélium) sont le tungstène thorié $\left(\mathrm{W}+\mathrm{ThO}_{2}\right)$ ou un alliage de tungstène-oxyde de lanthane $(\mathrm{W}+\mathrm{LaO})$ qui ont des températures de fusion élevée (de l'ordre de $3650 \mathrm{~K}$ ) [86]. L'ajout à ces cathodes chaudes des oxydes de thorium ou de lanthane fait baisser la température de surface de la cathode et réduit le potentiel d'extraction de ces matériaux [87]. Par ailleurs et dans le cas d'une atmosphère oxydante souvent des cathodes en zirconium ou hafnium sont utilisées [88]; compte tenu de leur faible conductivité thermique, ces matériaux doivent être utilisés suivant le dispositif de la pastille emboutie dans un matériau bon conducteur (cf. § 1.2.2.a). Le mécanisme de fonctionnement de ces métaux repose sur la création d'une pellicule oxydée sur la surface du métal, et qui a, par rapport à ce dernier, une température de fusion plus élevée. Cependant compte tenu de la faible durée de vie de ces cathodes (environ $20 \mathrm{~h}$ ) il est souvent préférable d'utiliser des cathodes en tungstène protégées du gaz oxydant par un faible écoulement de gaz non oxydant (moins de $5 \%$ de l'écoulement total suffit généralement) [89].

3.2.1 Gaz non oxydants. - a) Stabilisation par injection de gaz et parois froides (cathodes non noyées). - Ces générateurs à plasma se présentent généralement sous la forme classique d'une cathode en tungstène et d'une anode en cuivre qui sont refroidies par une circulation d'eau désionisée. La figure 20 suivante présente la conception d'après [90].

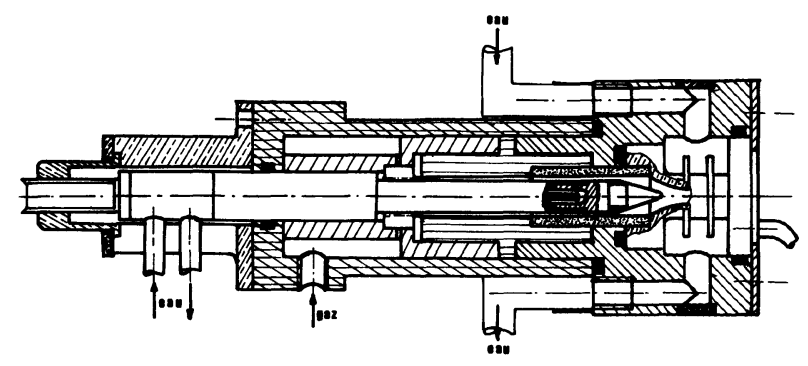

Fig. 20. - Générateur à plasma d'après [90].

[Plasma generator [90].] 
L'arc est créé entre la pointe cathodique et le canaltuyère anode, il est soufflé à l'extérieur où il forme le jet de plasma. Le gaz de fonctionnement (tel que l'azote, l'argon ou l'hydrogène) est introduit quelquefois axialement mais souvent tangentiellement créant une composante rotationnelle additive, assurant ainsi une meilleure stabilisation de l'arc et une rotation du pied de l'arc dans la région anodique. La figure 21 donne un exemple de l'évolution de la tension aux bornes de l'arc en fonction de l'intensité et pour différents gaz [91]. La température maximale obtenue est fonction des paramètres de fonctionnement du générateur, notamment le débit et la nature du gaz, elle peut atteindre $20000 \mathrm{~K}$ sur l'axe du jet de plasma ; la température moyenne est de l'ordre de 8000 à $12000 \mathrm{~K}$; le flux de chaleur étant lui de l'ordre de $7 \mathrm{~kJ} / \mathrm{cm}^{2} / \mathrm{s}$. Toutefois de tels jets de plasma sont généralement caractérisés par des gradients de température et de vitesse très importants (jusqu'à $4000 \mathrm{~K} / \mathrm{mm}$ et $200 \mathrm{~m} / \mathrm{s} / \mathrm{mm}$ sur le rayon) [92, 93]. Une augmentation du diamètre des tuyères se traduit par une baisse de la température et de la vitesse du gaz, ces baisses étant plus sensibles pour la vitesse que pour la

$$
\begin{gathered}
R e<110 \text { à } 250 \\
110 \text { à } 250<R e<500 \text { à } 750 \\
R e>500 \text { à } 750
\end{gathered}
$$

Dans un système laminaire (où en général le débit du gaz est faible) le niveau sonore est faible, le jet est très lumineux et peut atteindre des longueurs de $30 \mathrm{~cm}$ ou plus. Par contre le passage au régime turbulent augmente notablement la puissance acoustique et diminue la longueur du jet plasmagène à quelques $\mathrm{cm}$ [87].

Les rendements de ces générateurs varient selon le gaz employé (en général moins de $5 \%$ de l'énergie est perdue par refroidissement à la cathode et les pertes à l'anode varient entre 15 et $75 \%$ suivant la puissance et le débit de gaz). Par exemple :

$$
\begin{aligned}
& \eta=30 \text { à } 60 \% \text { pour } \mathrm{Ar} \\
& \eta=60 \text { à } 80 \% \text { pour } \mathrm{N}_{2} \text { et } \mathrm{H}_{2}
\end{aligned}
$$

ils sont très faibles en régime laminaire (moins de $20 \%$ ) quel que soit le gaz. La puissance de ces générateurs est généralement limitée à $100 \mathrm{~kW}$. Ils sont utilisés principalement pour la projection, la soudure et le découpage. Toutefois dans ces deux derniers cas on peut s'affranchir des limitations imposées par les paramètres opérationnels des générateurs à arc soufflé et augmenter considérablement la température moyenne du gaz et le flux de chaleur en utilisant des arcs transférés.

Le principe du transfert d'arc (Fig. 22) consiste à

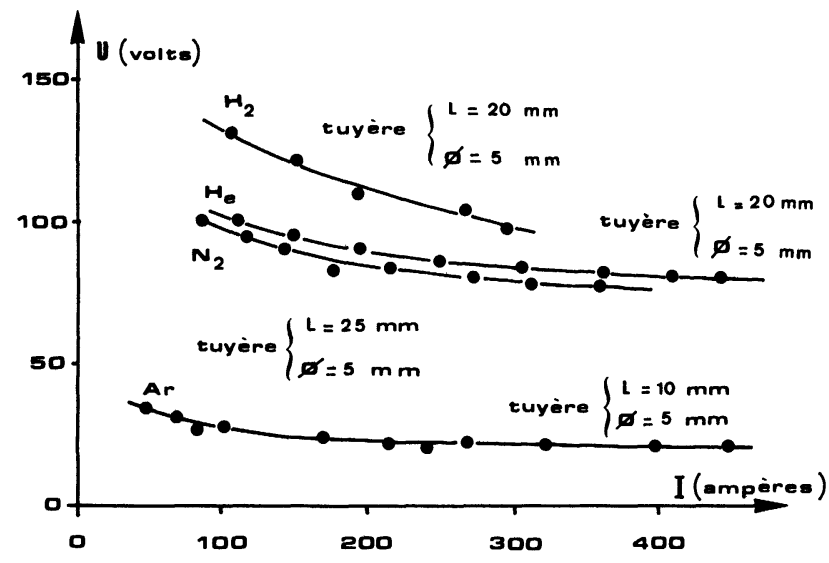

Fig. 21. - Influence de lintensité $I$ sur la tension $U$ aux bornes de l'arc pour des gaz différents $D_{\mathrm{G}}=30,4 \mathrm{l} / \mathrm{min}$. d'après [57].

[The influence of the intensity $I$ of the arc on the voltage $U$ for different gases $D_{\mathrm{G}}=30.4 \mathrm{l} / \mathrm{min}$. [57].]

température [94]. L'écoulement du jet obtenu peut être laminaire ou turbulent en passant par une forme transitoire. Les nombres de Reynolds définissant ces différentes formes sont d'après [87] :

\section{l'écoulement est laminaire \\ l'écoulement est transitoire \\ l'écoulement est turbulent .}

faire passer un courant de l'électrode interne d'un générateur à arc soufflé à une électrode externe constituée par la pièce à façonner (découper ou souder...) ou par un bain métallique.

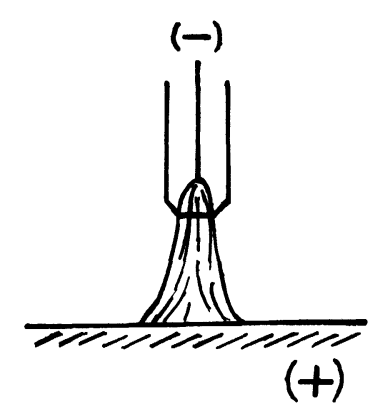

Fig. 22. - Principe d'arc transféré.

[Principle of a transferred arc.]

La tuyère forme un canal de guidage de la colonne de plasma produite et sert à la stabiliser. Dans cette configuration la puissance susceptible d'être dissipée est beaucoup plus élevée car la densité de courant sur la tuyère est nulle. La température maximale pouvant être atteinte est de l'ordre de $30000 \mathrm{~K}$, le flux de chaleur de l'ordre de $25 \mathrm{~kJ} / \mathrm{cm}^{2} / \mathrm{s}$ [95] et les puissances peuvent facilement dépasser $100 \mathrm{~kW}$. 
Une autre configuration permettant d'atteindre des puissances élevées avec ces générateurs à plasma est celle des arcs superposés [96].

Deux générateurs à plasma fonctionnant en courant continu et placés de telle façon que leurs jets concourent en un même point servent de conduction pour une superposition de puissance (Fig. 23) formant ainsi une colonne de plasma à température relativement élevée. Cette colonne joue le rôle d'une résistance gazeuse chauffant par rayonnement l'espace et la matière environnante. Les avantages de ce système sont les suivants :

- facilité de manipulation,

- possibilité d'atteindre de grandes puissances (2 à $300 \mathrm{~kW}$ ) en allongeant la colonne formée donc en augmentant la tension pour des intensités relativement moyennes,

- fonctionnement avec divers gaz selon l'application recherchée,

- rendement élevé atteignant $85 \%$.

Les difficultés d'amorçage de la superposition entre les deux jets pour des distances importantes forment le principal inconvénient de ce dispositif [97].

A titre d'exemple, nous donnons quelques caractéristiques de fonctionnement :

$$
\begin{aligned}
& \text { Générateur à plasma } \mathrm{A}: \\
& \text { débit d'argon }=12 \mathrm{l} / \mathrm{min} . \\
& V=22 \mathrm{~V} \quad I=600 \mathrm{~A} \quad P=13,2 \mathrm{~kW} .
\end{aligned}
$$

Générateur à plasma $\mathrm{B}$ : débit d'azote $=12 \mathrm{l} / \mathrm{min}$.

$$
V=70 \mathrm{~V} \quad I=300 \mathrm{~A} \quad P=21 \mathrm{~kW} .
$$

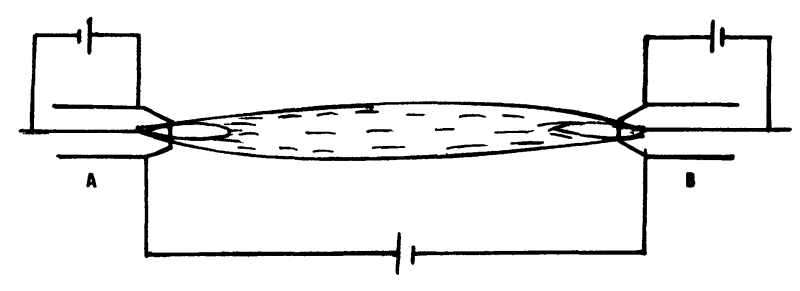

Fig. 23. - Principe de la superposition de puissance entre deux générateurs à plasma.

[Schematic of the power superposition between two plasma generators.]

\section{Superposition :}

$$
V=300 \mathrm{~V} \quad I=300 \mathrm{~A} \quad P=90 \mathrm{~kW} .
$$

Distance entre les deux générateurs $=42 \mathrm{~cm}$.

La puissance totale est donc de l'ordre de $125 \mathrm{~kW}$, et le rendement de la colonne de plasma atteint $84 \%$ [97].
Avec une telle configuration il est possible soit de fondre facilement des matériaux céramiques tels que l'alumine [98], la magnésie ou la zircone dans un four rotatif à axe horizontal [99], ou dans un four rotatif à axe vertical [100], soit de traiter des oxydes métalliques pour l'obtention de métaux à caractéristiques cherchées [39].

b) Stabilisation par injection tourbillonnaire de gaz (cathodes noyées). - Dès lors qu'il s'agit de faire fonctionner les genérateurs à plasma à des puissances élevées (supérieures à $150 \mathrm{~kW}$ ) ou même simplement à plus basse puissance avec des gaz oxydants (cathodes en zirconium ou hafnium), il convient d'assurer un très bon refroidissement des cathodes, la conductivité thermique des réfractaires (notamment $\mathrm{Zr}$ ) n'étant pas excellente. C'est pourquoi la technique consiste à les noyer complètement dans leur support en cuivre en ne laissant affleurer que l'extrémité du réfractaire et en lui donnant le plus petit diamètre possible compatible avec la tache cathodique requise pour la puissance demandée (par exemple $3 \mathrm{~mm}$ pour $p<80 \mathrm{~kW}$ ). Le problème est alors d'avoir une injection tourbillonnaire de gaz qui centre parfaitement la tache cathodique sur le réfractaire et évite l'accrochage de l'arc sur le cuivre.

La figure 24 présente un exemple d'application, ce générateur peut fonctionner à une puissance maximale de $500 \mathrm{~kW}$ [102].

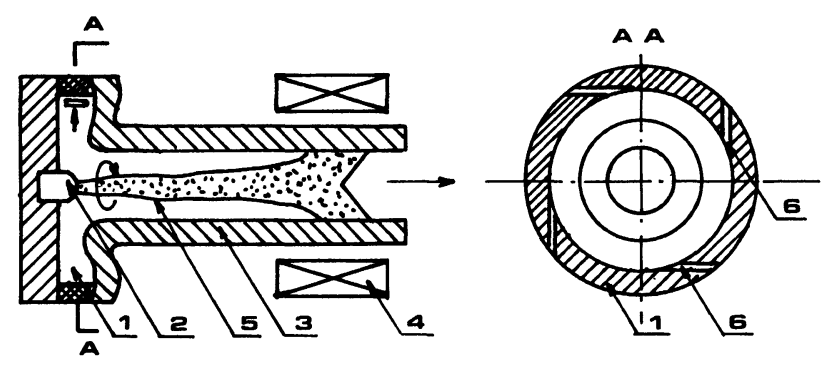

Fig. 24. - Générateur à plasma à injection tourbillonnaire.

1) Chambre de l'arc. 2) Cathode. 3) Anode. 4) Bobine magnétique. 5) Arc. 6) Entrées de gaz.

[Plasma generator with a vortex injection. 1) Arc chamber. 2) Cathode. 3) Anode. 4) Magnetic coil. 5) Arc. 6) Gas inlet.]

Le développement de tels arcs et la longueur qu'ils peuvent atteindre est alors conditionné par les phénomènes de turbulence. En effet comme on peut le voir sur la figure 25 on constate d'abord l'existence d'une zone d'arc très stable correspondant au développement mais au non mélange des couches limites thermiques chaudes autour de l'arc et froides autour de la paroi, dans la zone II ce mélange se fait et l'on voit apparaitre des turbulences qui deviennent très importantes dans la zone III pouvant entraîner l'accrochage erratique de l'arc. Le champ électrique augmente sensiblement dans la zone II et la quantité 
de chaleur perdue aux parois devient considérable dans la zone III. Il est donc nécessaire d'imposer à l'arc une longueur totale inférieure à celle des zones I et II afin de diminuer ces pertes. On peut aussi reculer la zone III par des injections multiples de gaz [101].
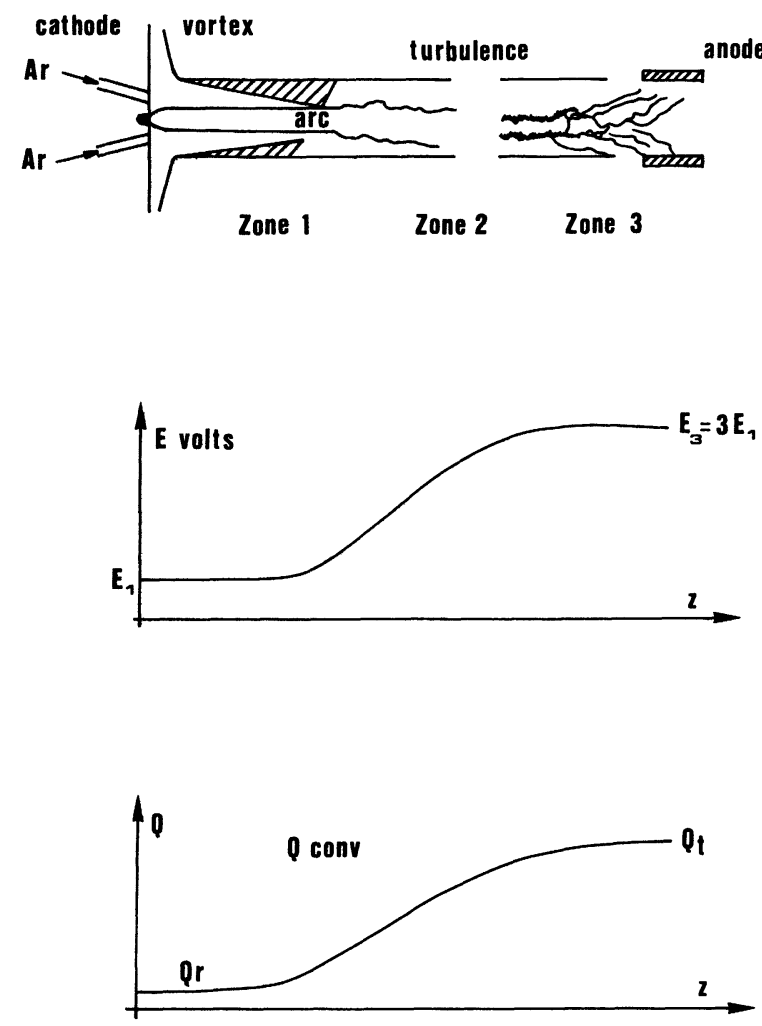

Fig. 25. - Développement d'un arc long, la variation du champ électrique et le flux de chaleur perdu.

[The development of a long arc, the variation of an electric field and the heat flux.]

Le gaz est injecté dans le constricteur pariétalement, et l'anode est protégée par un champ magnétique diminuant son érosion [101]. Les figures 26 et 27 présentent le schéma de ce type de générateur ainsi que quelques-unes de ces caractéristiques. Il est à noter que l'augmentation brusque du champ électrique $E$ indique une transition vers l'écoulement turbulent, s'accompagnant d'une augmentation du flux de chaleur à la paroi $\mathrm{Q}$.



Fig. 26. - Générateur à plasma à injection multiple d'après [101]. [Multiple injection plasma generator [101].]

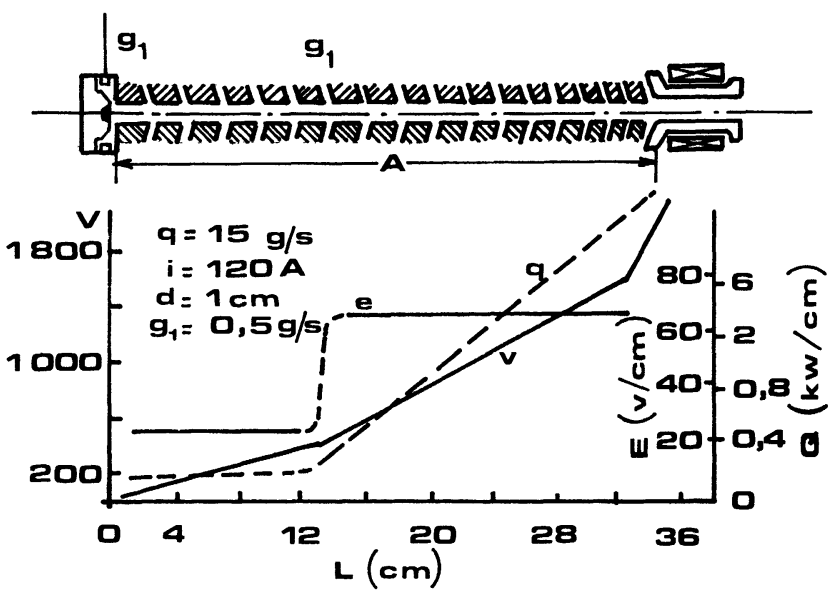

Fig. 27. - Caractéristiques d'un générateur à plasma à injection multiple d'après [101].

[The multiple injection plasma generator characteristics [101].]

Une relation semi-empirique présente cette variation pour l'air avec divers paramètres :

$$
E d=115(I / d)^{-0,23}(G / d)^{0,47}(p d)^{0,2}
$$

avec

$$
\begin{array}{ll}
I / d & \text { variant entre }(2 \text { et } 30) \times 10^{3} \mathrm{~A} / \mathrm{m} \\
G / d & \text { variant entre } 0,5 \text { et } 2 \mathrm{~kg} / \mathrm{s} \mathrm{m} \\
p d & \text { variant entre }(1 \text { et } 8) \times 10^{3} \mathrm{~N} / \mathrm{m}
\end{array}
$$

La puissance de fonctionnement de ce générateur à transpiration varie entre 1 et $2 \mathrm{MW}$.

Dans une telle configuration la stabilité de l'arc est assurée par le vortex créé par l'injection pariétale de l'air.

Une autre méthode consiste à utiliser une anode avec une brusque variation de diamètre (Fig. 28) entraînant, par effet aérodynamique, l'accrochage de l'arc dans la zone d'agrandissement de diamètre. En effet si la

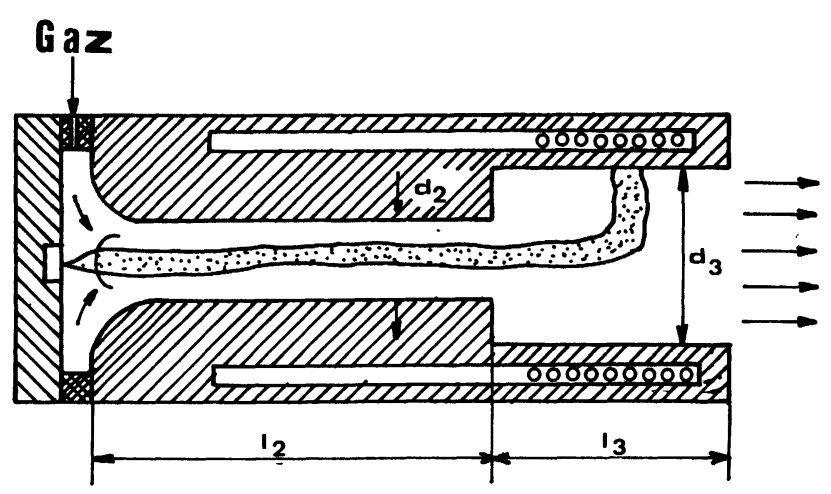

Fig. 28. - Générateur à plasma à changement de diamètre $(P=1 \mathrm{MW})$ d'après [102].

[Two diameters plasma generator $(P=1 \mathrm{MW})$ [102].] 


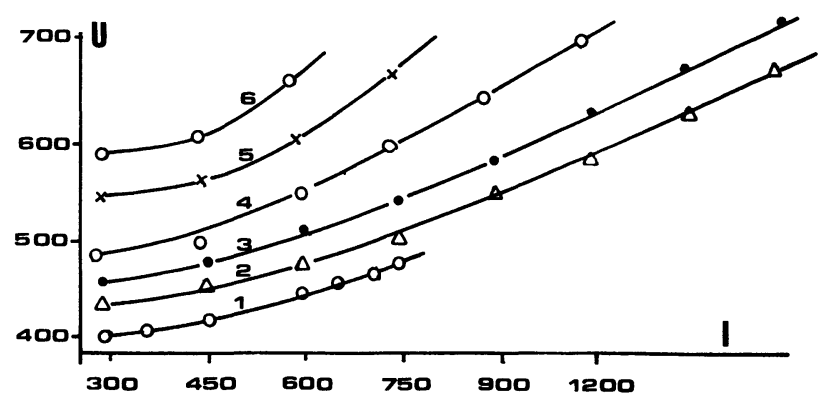

Fig. 29. - Caractéristiques montantes des générateurs à plasma à changement de diamètre [102].

$l_{2}=16,5 \times 10^{-2} \mathrm{~m} \quad d_{2}=3,0 \times 10^{-2} \mathrm{~m} \quad p=10^{5} \mathrm{~N} / \mathrm{m}^{2}$

1) $30 \times 10^{-3} \mathrm{~kg} / \mathrm{s} \quad$ 4) $120 \times 10^{-3}$

2) $60 \times 10^{-3} \quad$ 5) $150 \times 10^{-3}$

3) $90 \times 10^{-3} \quad$ 6) $180 \times 10^{3}$.

[The two diameters plasma generator characteristics [102].] longueur de la zone la plus constrictée (diamètre $d_{1}$ ) n'excède pas la longueur de la zone laminaire $I$ définie précédemment, le brusque changement de diamètre $d_{2}$ crée une zone turbulente dans le début du canal cylindrique refroidissant mieux celui-ci et amenant l'arc à s'accrocher plus loin dans une zone de l'anode à température plus élevée. Ceci permet, en respectant un rapport $d_{2} / d_{1}$ de 1,5 à 2 , d'avoir des longueurs $l$, de la zone plus constrictée assez importante et de travailler avec de fortes tensions. De plus on constate un accrochage de l'arc en parapluie de manière assez diffuse sur l'anode et donc une très bonne tenue de celle-ci. Enfin les caractéristiques de ces arcs sont montantes, ce qui est un avantage certain (Fig. 29) puisque cela permet de supprimer la résistance de stabilisation en série. Une relation semi-empirique corrèle la tension [102] aux différents paramètres géométriques représentés sur la figure précédente, elle est donnée pour l'air par :

$$
U=4,55\left(1+4,6 \times 10^{-5}\left(I / d_{2}\right)\right)\left(G / d_{2}\right)^{0,22}\left(l_{2} / d_{2}\right)^{0,95}\left(p d_{2}\right)^{0,23}
$$

avec

$$
\begin{array}{ll}
I / d_{2} & \text { variant entre }(0,8 \text { et } 4) \times 10^{4} \mathrm{~A} / \mathrm{m} \\
G / d_{2} & \text { variant entre }(0,8 \text { et } 6,5) \mathrm{kg} / \mathrm{s} \mathrm{m} \\
l_{2} / d_{2} & \text { variant entre }(5,6 \text { et } 14,5) \\
p d_{2} & \text { variant entre }(2 \text { et } 40) \times 10^{3} \mathrm{~N} / \mathrm{m} .
\end{array}
$$

Ces générateurs avec tuyères à changement de diamètre, peuvent fonctionner à des puissances de l'ordre de $1 \mathrm{MW}$.

c) Stabilisation par champ magnétique.-Plusieurs générateurs à plasma utilisent la configuration décrite au paragraphe 1.3. Une étude de l'écoulement du plasma dans une tuyère en présence d'un champ magnétique axial ainsi que la conception de ce genre de générateur sont présentées par [103], la figure 30 montrant schématiquement le générateur utilisé. Il peut fonctionner à l'argon avec une puissance atteignant $20 \mathrm{~kW}$ avec un champ magnétique maximal de $7000 \mathrm{G}$ et une enthalpie spécifique de $10000 \mathrm{~kJ} / \mathrm{kg}$. Il est à noter que généralement le champ magnétique représente nettement moins de $10 \%$ de la puissance fournie à ces générateurs.

Un champ magnétique est souvent rajouté autour des électrodes des générateurs à plasma classiques $(\S 3.2 .1 . a)$ à électrodes coaxiales. Plusieurs travaux ont porté sur ce type de générateur. La figure 31 montre le schéma de principe de ce générateur à arc magnétisé d'après [104].

3.2.2 Gaz oxydants. - Les différents générateurs à plasma que nous venons de présenter fonctionnent avec des gaz tels que l'argon, l'azote, l'hélium ou l'hydrogène qui ne réagissent pas chimiquement avec

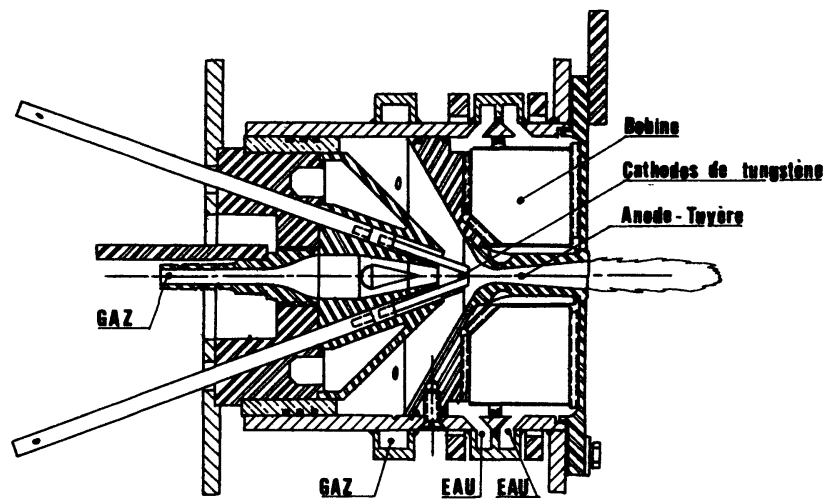

Nivcuirre rouge

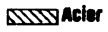

Iselant

Fig. 30. - Coupe longitudinale du générateur à plasma à champ magnétique d'après [103].

[Magnetic field plasma generator [103].]

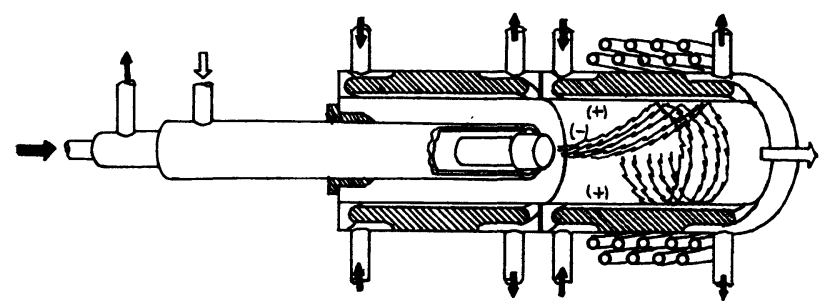

Fig. 31. - Générateur à plasma à champ magnétique d'après [104]. [Magnetic field plasma generator [104].] 
les matériaux constituant les électrodes (surtout les cathodes réfractaires chaudes). Cependant l'utilisation de gaz oxydants (l'air par exemple présente un grand intérêt économique et des applications spécifiques recherchées tels que le réchauffage, la production des oxydes d'azote, ou l'oxydation des halogénures métalliques).

Différents générateurs à plasma ont donc été adaptés au fonctionnement sous atmosphère oxydante soit par l'utilisation des cathodes spéciales en zircone ou zirconium, soit en assurant une protection par l'utilisation d'un petit débit de gaz neutre.

a) Générateurs à cathode en $\mathrm{Zr}$. - Le générateur à plasma classique mis au point à Limoges et déjà décrit $(\S 3.2 .1 . a)$ a été adapté au fonctionnement sous atmosphère oxydante [105]. La cathode est alors constituée d'un petit barreau de zirconium de diamètre $4 \mathrm{~mm}$ noyé (emmanchement à force) dans un $1^{\mathrm{er}}$ porte-cathode en cuivre lui-même centré et vissé sur un $2^{\mathrm{e}}$ porte-cathode refroidi par circulation d'eau (Fig. 32).

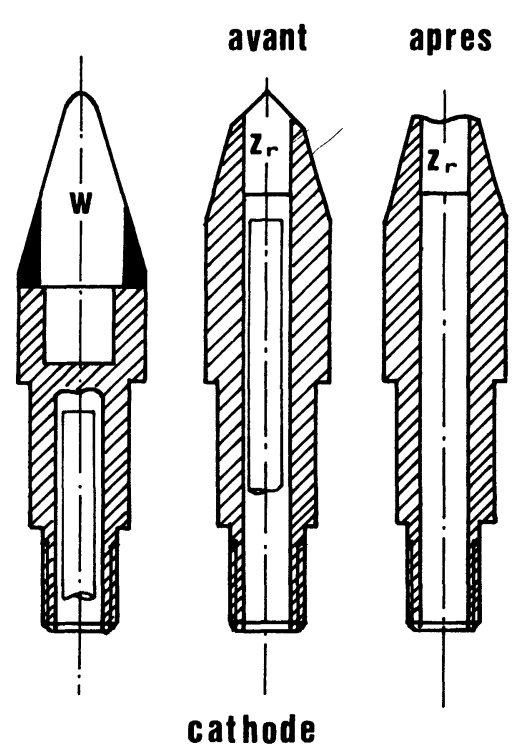

Fig. 32. - Cathode en $\mathrm{Zr}$ fonctionnant sous atmosphère oxydante. [ $\mathrm{Zr}$ cathode working in oxidizing atmosphere.]

Dès l'amorçage de l'arc, le zirconium atteint sa température de fusion $(2130 \mathrm{~K})$ et réagit lors de l'injection de l'oxygène pour recouvrir la cathode d'une pellicule d'oxyde $\mathrm{ZrO}_{2}$. Le zircone, par émission thermo-ionique [88], est un émetteur d'électrons et constitue donc un point d'accrochage préférentiel de l'arc.

La figure 33 représente les caractéristiques de fonctionnement courant-tension pour différents débits d'air. Pour des débits de 0,8 et $2,1 \mathrm{~g} / \mathrm{s}$, ces caractéristiques sont normalement descendantes, par contre à fort débit $3,1 \mathrm{~g} / \mathrm{s}$ l'arc est soufflé à l'extérieur du canal de la tuyère et vient s'accrocher sur la face externe de l'anode; le comportement de l'arc est alors imposé

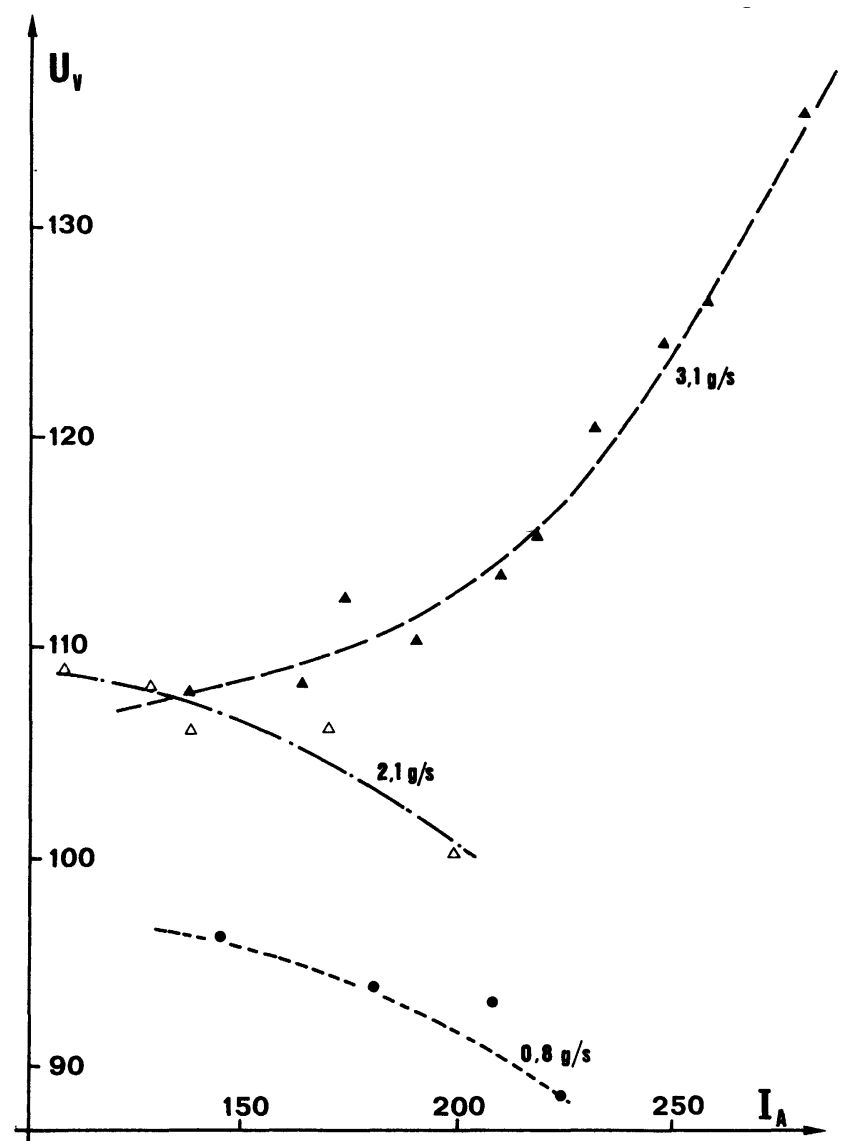

Fig. 33. - Caractéristiques courant-tension pour différents débits d'air d'après [90].

[The characteristics (intensity, voltage) for different mass flow rates [90].]

par la géométrie de la tuyère et la caractéristique est montante [90].

Un dispositif similaire est utilisé dans les générateurs russes décrits au paragraphe 2.2.1.b.

Toutefois il faut noter que la durée de vie moyenne de telles cathodes ne dépasse pas $20 \mathrm{~h}$ et que le réamorçage de l'arc, du fait de la pellicule de $\mathrm{ZrO}_{2}$ isolante à froid, est difficile. L'arc doit alors s'amorcer sur le cuivre afin de fondre la pellicule de $\mathrm{ZrO}_{2}$ et l'on détériore facilement le porte-cathode. C'est pourquoi on utilise de préférence des cathodes en tungstène protégées [102] par un écoulement (moins de $10 \%$ de l'écoulement total) de gaz non oxydant ( $\mathrm{Ar}$ ou $\mathrm{N}_{2}$ généralement) (cf. Fig. 34). Le débit relatif de gaz protecteur est d'ailleurs fonction du diamètre et de la longueur du canal cylindrique disposé devant la cathode et percé dans la paroi séparant les deux écoulements.

b) Générateurs à cathode en $\mathrm{ZrO}_{2}$. - Une cathode en oxyde réfractaire (en zircone) a été utilisée par [106-108] au C.N.R.S. d'Odeillo sous une atmosphère oxydante. Le schéma de l'appareillage (Fig. 35) montre l'anode tubulaire métallique (cuivre) refroidie par eau, le support de la cathode constitué d'un creuset 


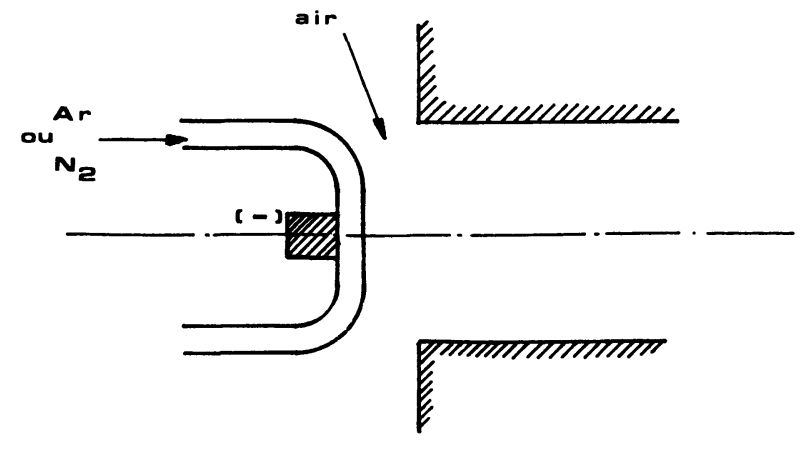

Fig. 34. - Principe des cathodes noyées et protégées par un écoulement gazeux.

[The principle of the immerged cathodes with gaz flow protection.]

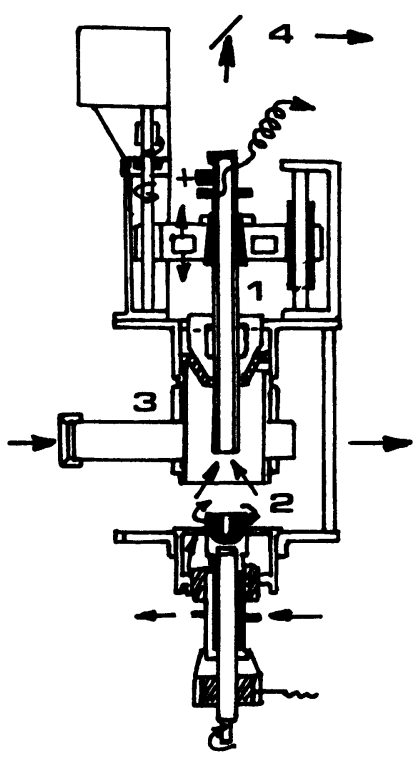

Fig. 35. - Schéma de l'appareillage d'étude d'après [107].

[Schematic arrangement of the apparatus [107].]

en acier inoxydable refroidi par circulation d'eau et muni d'une pointe en molybdène assurant le contact entre l'oxyde et le circuit électrique (il est entraîné en rotation par un moteur), ainsi que l'enceinte étanche contenant l'ensemble. La poudre de $\mathrm{ZrO}_{2}$ est tassée dans le creuset métallique. A la suite de l'amorçage par une tige, l'oxyde fond et recouvre la pointe de Mo, grâce à la centrifugation le liquide prend la forme d'une cavité de géométrie stable pseudoparabolique. La surface interne de la cavité joue alors le rôle de cathode pour le maintien de l'arc. La puissance de fonctionnement de ce générateur est de l'ordre de $10 \mathrm{~kW}$ avec des débits de gaz de quelques dizaines de litres/minute.

3.2.3 Les liquides. - Partant de l'arc de Gerdien [109] repris par Maecker [19], Weiss [110] a développé le premier type de générateur à eau schématisé sur la figure 36 . La tuyère en graphite sert de cathode et afin d'éviter qu'elle ne soit couverte d'eau, son diamètre est inférieur à celui du tourbillon d'eau. Toute

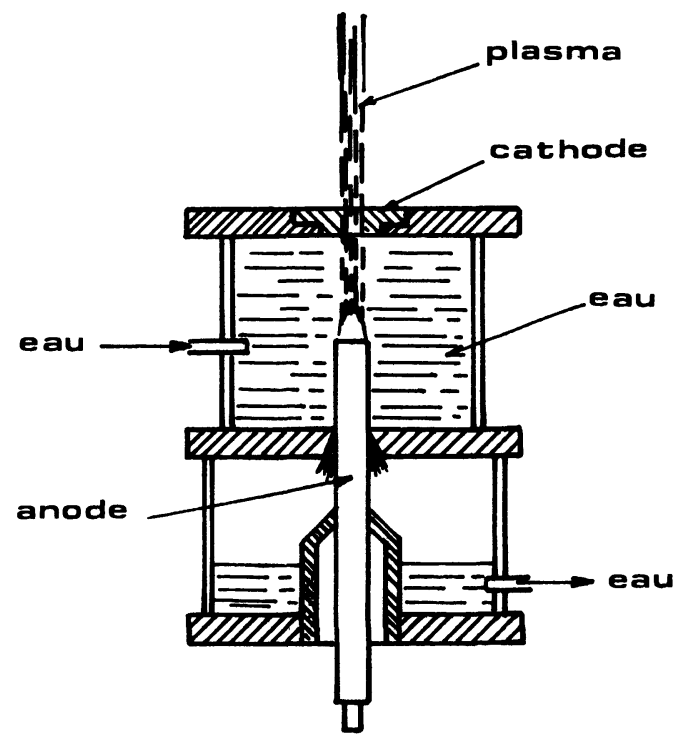

Fig. 36. - Schéma de principe du générateur à plasma stabilisé par écoulement d'eau d'après [110].

[Wall stabilized plasma generator [110].]

l'eau d'alimentation s'évacue entre l'anode centrale et la tuyère arrière. L'usure de la cathode ne permet pas un fonctionnement supérieur à quelques secondes et ce type d'appareil a été utilisé pour l'étude des conditions de re-entrée des missiles balistiques.

Les appareils développés à l'Institut de Recherche Tchécoslovaque pour les Industries Electriques (RIEE) ont repris [111] le principe du générateur de Weiss en tenant compte des impératifs suivants.

La conception doit permettre l'existence de deux tourbillons d'eau de diamètres différents; un petit diamètre pour le canal de stabilisation et un plus grand autour de l'électrode centrale.

Les parties exposées à la chaleur du plasma doivent être protégées par un film d'eau.

L'évacuation arrière doit être telle que le débit d'eau permette un refroidissement suffisant. La figure 37 représente un générateur fonctionnant à l'eau dont la construction respecte ces impératifs, la ligne en pointillés représentant le diamètre du tourbillon. L'eau est introduite tangentiellement dans deux chambres A et $B$ séparées par une tuyère intermédiaire isolée 1 de diamètre égal à celui de la tuyère anode. L'injection d'eau dans la chambre B par 2 permet de recouvrir entièrement l'électrode centrale d'un film d'eau protecteur. Le démarrage de l'arc est facilité par la soupape 3 dont la position basse permet au départ l'évacuation latérale de l'eau en 4 puis dès que l'arc fonctionne la position haute force l'évacuation de l'eau par le canal 5 le long de la cathode. Ces arcs ne peuvent être démarrés en haute fréquence et le départ est assuré par la mise en contact des électrodes par un fil ou un ruban métallique qui ne doit pas perturber l'écoulement. Enfin un système d'avance automatique de la cathode doit être prévu pour compenser son usure. 


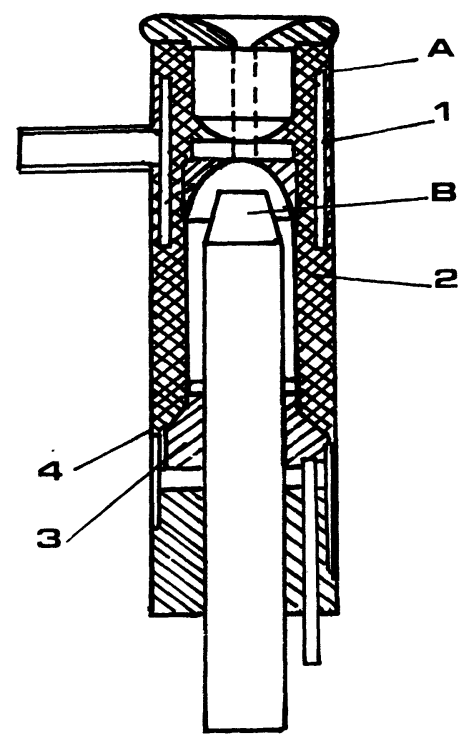

Fig. 37. - Générateur à plasma stabilisé par eau d'après [111]. [Wall stabilized plasma generator [111].]

Etant donné le prix de revient très faible, le contrôle aisé et la disponibilité pratiquement en tous lieux du fluide de stabilisation les générateurs à eau sont utilisés fréquemment dans l'industrie et en particulier dans les pays de l'Est. Gross [112] donne une description détaillée de nombreux générateurs de ce type.

Par ailleurs la société suisse LONZA a développé un générateur de $250 \mathrm{~kW}$ de puissance (Fig. 38) fonctionnant à l'alcool ou à l'eau [113]. La vitesse de rotation du liquide crée un vortex stabilisant l'arc. Une faible quantité de liquide est vaporisée de la surface interne du vortex et forme le plasma. La cathode est en graphite qui n'est que très faiblement consommé et donc avancé régulièrement. L'anode est un disque de cuivre refroidi par eau et qui du fait d'une vitesse de rotation (3000 t/min.) a une durée de vie de plusieurs centaines d'heures. Des rendements thermiques de $90 \%$ sont donnés pour des puissances

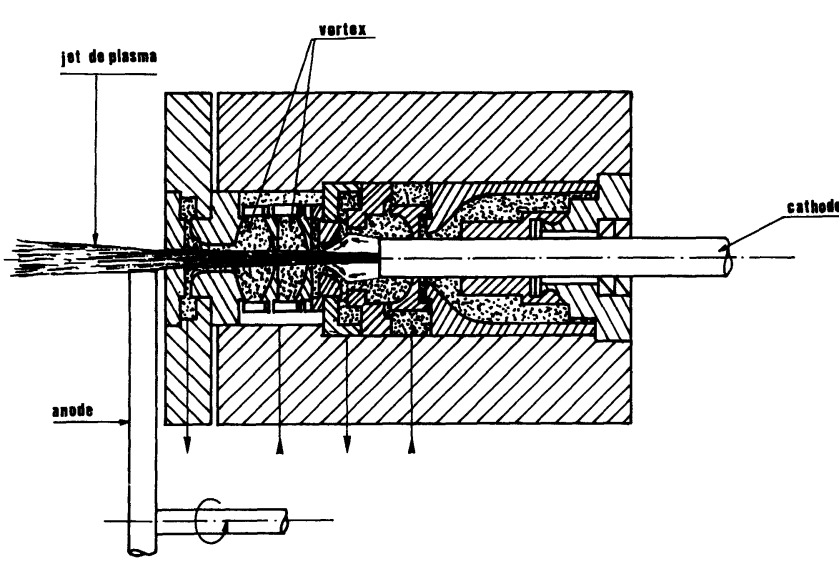

Fig. 38. - Torche à plasma stabilisée par un liquide d'après [113]. [Liquid stabilized plasma generator [113].] de $200 \mathrm{~kW}$ avec des liquides tels que le mélange eauméthanol ou méthanol. Ce générateur est utilisé industriellement pour la projection ou la sphéroïdisation de divers matériaux.

3.3 GÉNÉRATEURS À ÉleCTRODES froides. - Dans le chapitre précédent, nous avons passé en revue les caractéristiques de quelques générateurs à plasma ayant leur cathode qui atteint des températures élevées (mécanisme d'émission thermo-électronique prédominant). Nous allons maintenant indiquer les caractéristiques de quelques générateurs à électrodes froides et dans lesquels la zone radiale de l'arc se déplace sous l'action des forces aérodynamiques et/ou électromagnétiques.

Notons que le pied de l'arc se déplace par à-coups. Dans l'intervalle entre les sauts, les micropointes de ces attachements diffus restent immobiles pendant $10^{-4}$ à $10^{-5} \mathrm{~s}$, et seule une couche mince de la surface du métal a le temps de chauffer. Le temps d'existence de ces micropointes au même endroit est déterminé par différents facteurs notamment la température de la surface, sa rugosité, le degré de turbulence du courant, la présence d'actions motrices sur la zone radiale de l'arc, etc...

3.3.1 A champ magnétique. - Un générateur à arc industriel fonctionnant chez Westinghouse aux U.S.A. en courant alternatif monophasé est présenté sur la figure 39 [114]. Cette unité peut atteindre 1,5 MW et pour une installation triphasée utilisant trois générateurs une puissance de l'ordre de 4,5 MW. Les électrodes de ces générateurs sont refroidies par circulation d'eau, un champ magnétique à courant continu assure la rotation des pieds de l'arc $(1000 \mathrm{t} / \mathrm{s})$ ainsi que l'injection tourbillonnaire de gaz (air par exemple) par une fente entre les deux électrodes ce qui a également pour effet de souffler l'arc dans la chambre.

La figure 40 montre les caractéristiques de ce type de générateurs fonctionnant avec de la vapeur d'eau et pour une intensité du courant fixée. Une enthalpie de l'ordre de $2,8 \times 10^{4} \mathrm{~kJ} / \mathrm{kg}$ peut être facilement atteinte à la sortie de la tuyère.

Une autre configuration utilisée par [101] en URSS est présentée sur la figure 41. Des bobines de champ magnétique assurent la protection des électrodes tubulaires refroidies au niveau des zones d'accrochage. Des injections de gaz à l'amont et à l'aval de la cathode assurent un vortex important renforçant la stabilité de l'arc. Une relation semi-empirique est donnée qui corrèle la tension avec les paramètres $I^{2} / G d, G / d$ et $p d$ dans une large gamme de valeurs; elle prend pour l'air la forme suivante :

$$
U^{+}=1360\left(I^{2}(G d)\right)^{-0,2}(G / d)^{0,25}(p d)^{0,35}
$$

avec

$I$ intensité du courant variant de 50 à $5000 \mathrm{~A}$

$G$ débit massique de gaz variant de $10^{-3}$ à $3,5 \mathrm{~g} / \mathrm{s}$

$d$ diamètre variant de 5 à $76 \mathrm{~mm}$

$p$ pression variant de $(1$ à 100$) \times 10^{5} \mathrm{~Pa}$ 


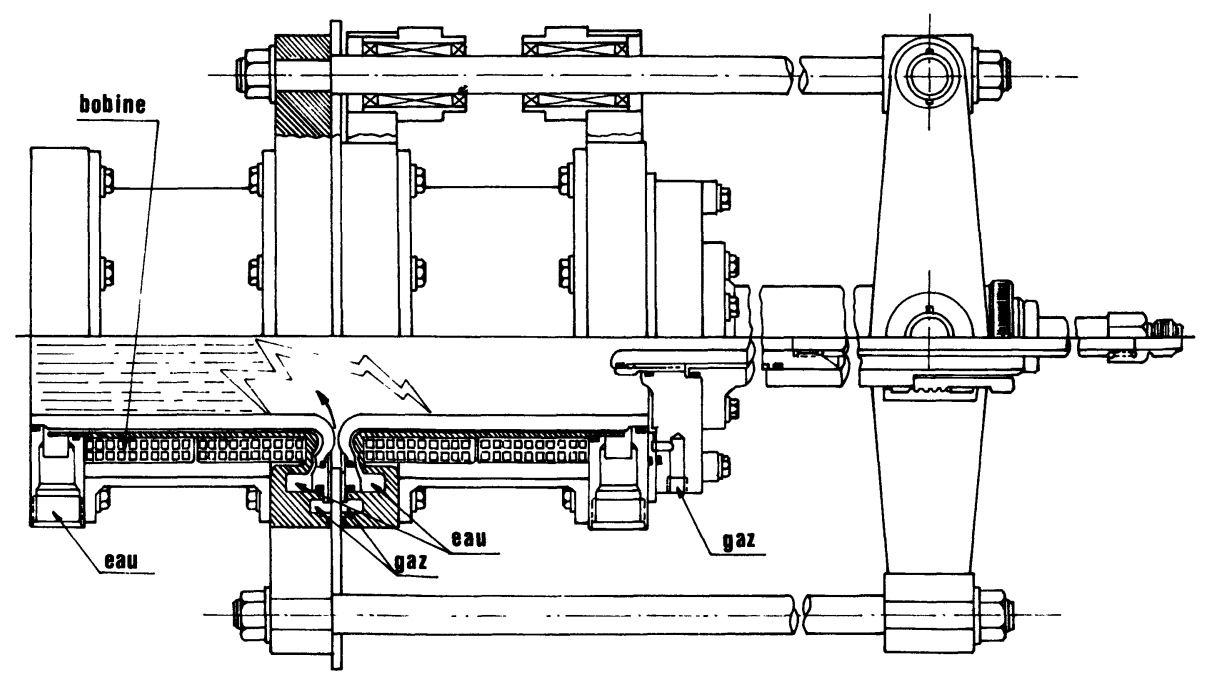

Fig. 39. - Générateur à plasma à électrodes froides et à champ magnétique [114].

[Cold electrodes and magnetic field plasma generator [114].]

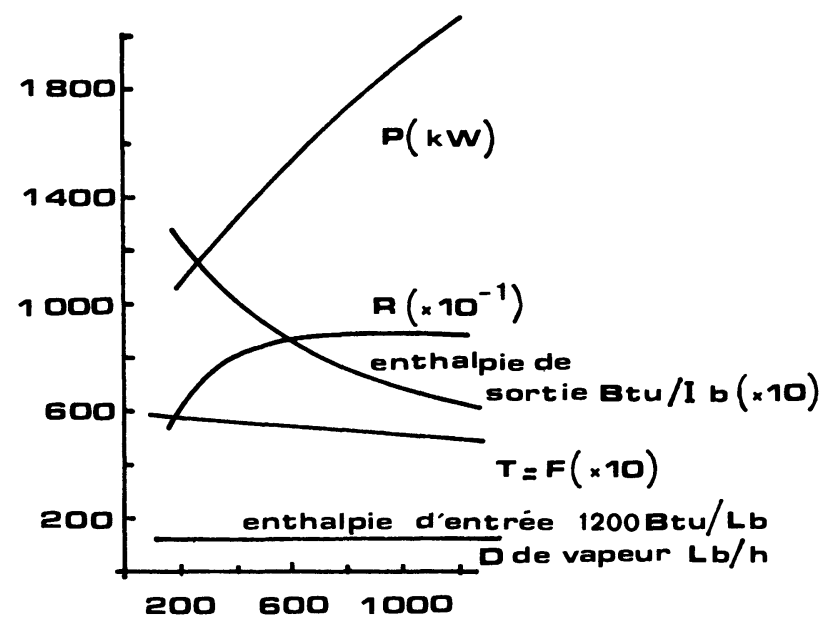

Fig. 40. - Caractéristiques des générateurs à plasma à électrodes froides et champ magnétique fonctionnant avec de la vapeur d'eau d'après [114].

[Plasma generator performance characteristics working with steam [114].]

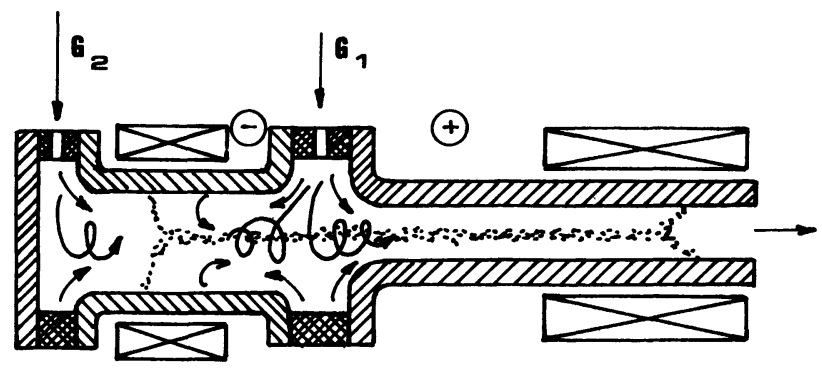

Fig. 41. - Générateur à plasma à deux chambres stabilisé par vortex et champ magnétique $(P=1$ à $1,5 \mathrm{MW})$ [101].

[Two-chambers plasma generator stabilized by the vortex and the magnetic field $(P=1$ to $1.5 \mathrm{MW})[101]$. elle est représentée sur la figure 42 pour un fonctionnement à l'air avec les valeurs expérimentales de plusieurs instituts en URSS qui ont servi à l'établir.

Ce générateur à deux chambres fonctionne à des pressions variant de 1 à $1,5 \mathrm{MW}$ et avec des enthalpies de l'ordre de (15 à 20$) 10^{3} \mathrm{~kJ} / \mathrm{kg}$.

Cette même configuration est aussi utilisée en courant alternatif [102], la relation semi-empirique donnant la valeur de la tension avec les différents paramètres pour l'air prend la forme :

$$
U=2150\left(I^{2} /(G d)\right)^{-0,16}(G / d)^{0,16}(p d)^{0,20} .
$$

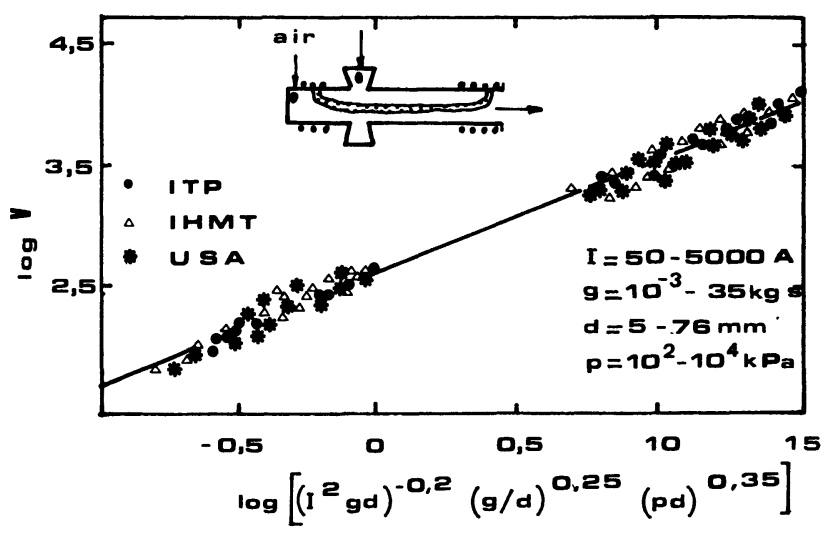

Fig. 42. - Corrélation semi-empirique pour des générateurs à arc à électrode froide stabilisés par vortex et champ magnétique et fonctionnant à l'air (ITP = Institut de Physique Thermique, Novossibirsk URSS $;$ IHMT $=$ Institut de Transfert de chaleur et de Masse. Minsk ('RSS) [102].

[Semi-empirical correlation for a vortex and magnetic field stabilized cold-cathode, arr plasma generator (ITP $=$ Institute of Thermal Physics, Novossibirsk USSR ; IHMT = Institute of Heat and Mass Transfer, Minsk USSR) [102].] 
3.3.2 A jets opposés. - Une configuration produisant deux jets de plasma simultanés a été développée par [115] en URSS (Fig. 43). Ces deux jets sont soufflés des deux côtés opposés du générateur par le gaz neutre ou oxydant (l'air). Chacune des deux électrodes froides est composée de deux parties cylindriques refroidies avec un changement de diamètres afin d'allonger l'arc (cf. § 3.2.1.b). La puissance pouvant être développée varie entre 200 et $3000 \mathrm{~kW}$.



Fig. 43. - Générateur à plasma à jets opposés d'après [115].

[Two opposite-jets plasma generator [115].]

Les avantages de ce genre de générateurs à plasma sont :

- une simplicité de construction,

- une durée de vie et de fonctionnement longue (avec les gaz nón oxydants la durée de vie des électrodes atteint $500 \mathrm{~h}$ ),

- une tension de fonctionnement élevée,

- la possibilité d'utilisation d'une source de courant électrique commune sans avoir recours à une stabilisation spéciale du courant.

Les caractéristiques de fonctionnement tensioncourant sont ascendantes (Fig. 44). Elles sont données pour un débit d'air variable de 100 à $356 \mathrm{~g} / \mathrm{s}$ et pour une géométrie déterminée.

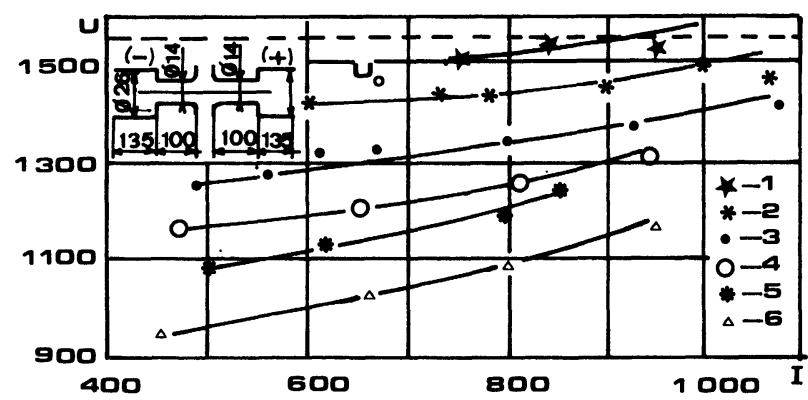

Fig. 44. - Caractéristiques de fonctionnement $U(I)$ des générateurs à jets opposés pour les débits d'air suivant.

$\nabla \begin{array}{lll}1 & 356 \mathrm{~g} / \mathrm{s}\end{array}$

○ $2300 \mathrm{~g} / \mathrm{s}$

- $3250 \mathrm{~g} / \mathrm{s}$

$\times 4200 \mathrm{~g} / \mathrm{s}$

$\triangle 5150 \mathrm{~g} / \mathrm{s}$

$\square 6 \quad 100 \mathrm{~g} / \mathrm{s}$.

[The two opposite-jets plasma generator characteristics for the following air flow rate.]

3.3.3 A électrode tubulaire fermée. - Les caractéristiques tension-courant d'un arc dans un générateur à plasma à électrodes cylindriques dont l'une est fermée d'un côté, ne donnant donc qu'un seul jet de plasma [116] et fonctionnant à différents gaz azote, argon, oxygène et hélium sont données sur la figure 45 .

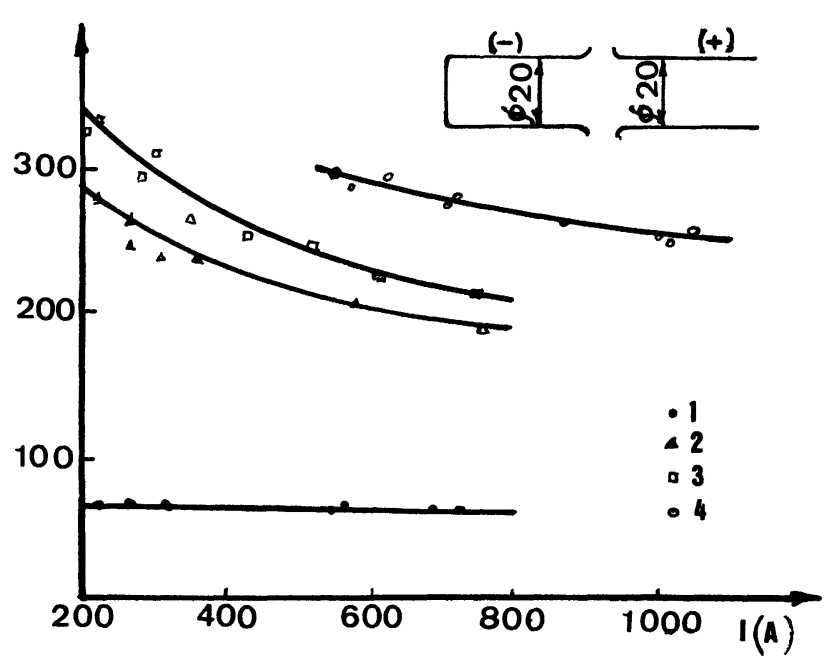

Fig. 45. - Caractéristiques de fonctionnement des électrodes tubulaires fermées pour un débit massique de $40 \mathrm{~g} / \mathrm{s}$ et pour les gaz suivants : 1 argon, $\triangle 2$ oxygène, $\square 3$ azote, $\bigcirc 4$ hélium d'après [116].

[Closed-tubular electrodes characteristics for a mass flow rate $=40 \mathrm{~g} / \mathrm{s}$ and for the following gases :

- 1 argon, $\Delta 2$ oxygen, $\square 3$ nitrogen, $\bigcirc 4$ helium after [116].]

Ces caractéristiques sont corrélées par l'équation empirique suivante contenant des nombres adimensionnels et présentées sur la figure 46 :

$$
\frac{U d \sigma_{0}}{I}=3,39\left(\frac{I^{2}}{G d \sigma_{0} h_{0}}\right)^{-0,62}
$$

avec

$U$ tension aux bornes de l'arc $(\mathrm{V})$

I intensité (A)

$d$ longueur (m)

$\sigma_{0}$ conductivité électrique de référence $\mathrm{mho} / \mathrm{m}$

$G$ débit massique de gaz $\mathrm{kg} / \mathrm{s}$

$h_{0} \quad$ l'enthalpie de référence $\mathrm{kcal} / \mathrm{kg}$.

3.3.4 "Gainage » par le gaz. - Un dispositif comprenant un générateur à plasma pilote classique (§ 3.2.1.a) et trois électrodes gainées à axes concourants disposées symétriquement par rapport à laxe du générateur pilote (Fig. 47) est développé par [117]. Les électrodes en cuivre refroidies reçoivent une alimentation en gaz plasmagène les gainant et les protégeant de l'usure, elles sont connectées aux bornes d'une source de courant alternatif triphasé.

Dès la production du jet de plasma pilote, lorsque les pointes des électrodes sont baignées par ce dernier, on observe le passage du courant électrique entre les électrodes. Chaque courant gazeux issu d'une électrode est alors transformé en jet de plasma et un écoulement 


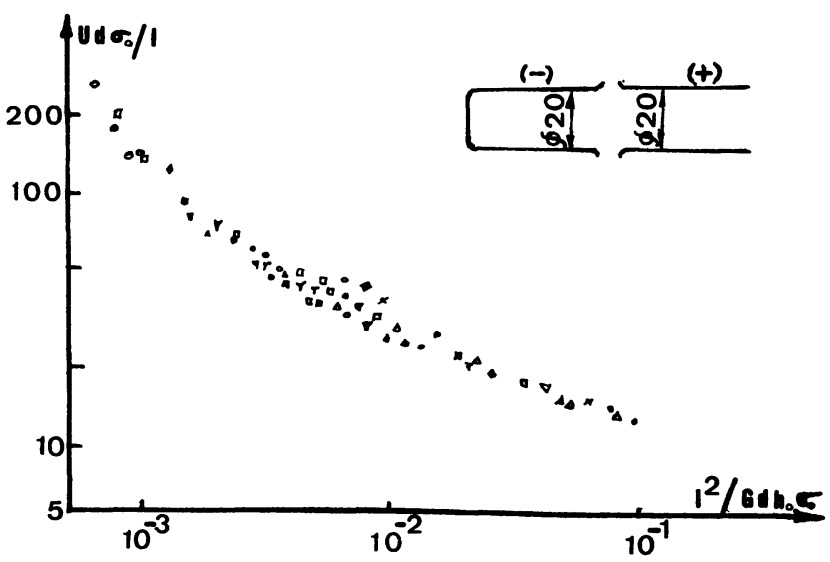

Fig. 46. - Caractéristiques généralisées des électrodes tubulaires fermées pour différents débits et différentes natures de gaz d'après [116].

Argon [Argon] : $4 \mathrm{~g} / \mathrm{s}: \Delta 6 \mathrm{~g} / \mathrm{s}: \nabla 8 \mathrm{~g} / \mathrm{s}: \Delta 12 \mathrm{~g} / \mathrm{s}$. Hélium [Helium] : $1 \mathrm{~g} / \mathrm{s}: \bigcirc 2 \mathrm{~g} / \mathrm{s}: \Delta 4 \mathrm{~g} / \mathrm{s}$. Oxygène [Oxygen] : $\square 4 \mathrm{~g} / \mathrm{s}$ : - $6 \mathrm{~g} / \mathrm{s}: \diamond 8 \mathrm{~g} / \mathrm{s}$. Azote [Nitrogen] : × $2 \mathrm{~g} / \mathrm{s}: 84 \mathrm{~g} / \mathrm{s}: \dot{\gamma} 6 \mathrm{~g} / \mathrm{s}$. [Generalized characteristics for the closed-tubular electrodes for the following mass flow rate and gases [116].]

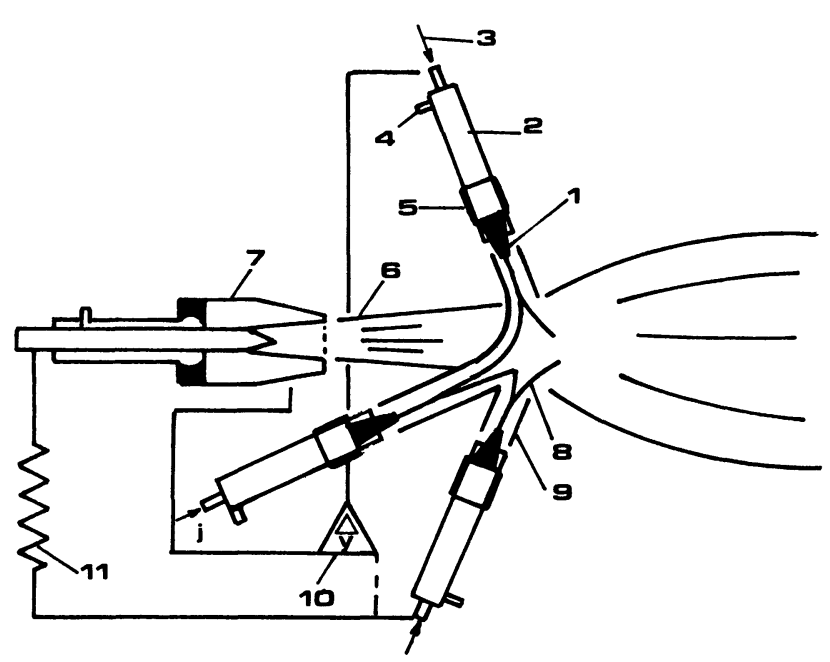

Fig. 47. - Dispositif à 3 électrodes mobiles d'après [117].

1) électrode; 2) support d'électrode; 3) vérin à double effet ; 4) entrée de gaz; 5) tube de gainage des gaz; 6) jet de plasma pilote ; 7) chalumeau à plasma pilote; 8) arc gainé : 9) tube : 10) source de courant triphasé : 11) résistance.

[Three moving electrodes [117].

1) electrode; 2) electrode support ; 3) pneumatic jack ; 4) gas inlet; 5) gas sheath tube ; 6) pilot plasma jet; 7) pilot plasma generator ; 8) sheathed arc ;9) tube ; 10) alternative current source : 11) resistance.]

permanent de plasma représentant la somme des écoulements produits par chaque électrode est alors créé.

Le chalumeau pilote est éteint dès l'amorçage du plasma principal. Le champ électrique est approximativement constant le long de la partie individualisée de chaque jet de plasma et ne dépend, à intensité constante, que du débit de gaz plasmagène. Une relation donnant la tension de fonctionnement $V$ (en volts) mesurée entre une électrode et le neutre est exprimée en fonction du débit de gaz $Q$ (en $\mathrm{g} / \mathrm{s}$ ) et de la distance inter-électrode $d(\mathrm{en} \mathrm{cm})$ sous la forme :

$$
V=0,5 Q d-4,85 Q+3,6 d+66
$$

pour une intensité efficace de $200 \mathrm{~A}$ [118].

Le rendement de ce dispositif est compris entre 90 et $95 \%$, sa puissance peut atteindre $200 \mathrm{~kW}$ et l'écoulement obtenu une longueur de $1 \mathrm{~m}$ et un diamètre de $25 \mathrm{~cm}$ pour un débit de $5 \mathrm{~g} / \mathrm{s}$ d'azote par exemple [118].

Ce type de réacteur est utilisé pour le traitement des matériaux solides qui sont injectés facilement dans l'axe du système (sphéroïdisation par exemple) [119, 120]. En utilisant des électrodes de carbone avancées en continu pour compenser leur usure, ce dispositif peut fonctionner à l'air.

4. Générateurs à forte enthalpie. - Les générateurs à forte enthalpie utilisés notamment pour la simulation aérodynamique fonctionnent à des enthalpies et des pressions élevées. L'enthalpie générée peut atteindre $10^{8} \mathrm{~kJ} / \mathrm{kg}$ avec des pressions allant jusqu'à $10^{4} \mathrm{kPa}$.

Le rendement thermique n'a qu'une faible importance dans ces applications, et le temps de fonctionnement est de l'ordre de quelques secondes ou tout au plus de quelques minutes.

Nous présentons par la suite quelques-uns de ces générateurs classés selon les modes de stabilisation utilisés.

4.1 GÉNÉRATEURS STABILISÉS PAR PAROI SEGMENTÉE FROIDE. - Le schéma de la figure 48 présente les principes de conception de ces générateurs à enthalpie élevée et à vitesse supersonique utilisés par la NASA $[121,122]$.

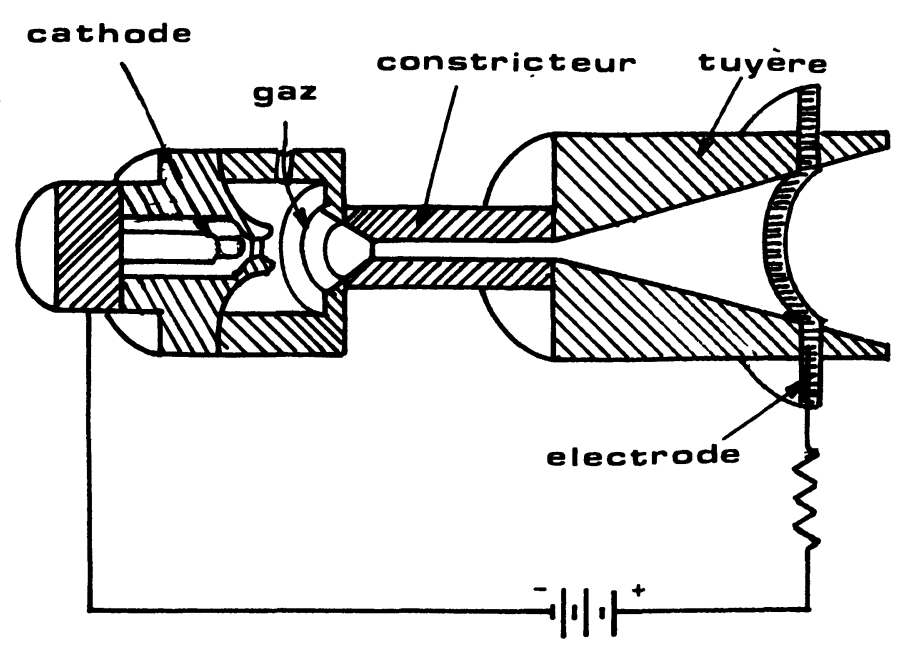

Fig. 48. - Générateur à forte enthalpie et vitesse supersonique utilisé par la NASA [122].

[High enthalpy and supersonic plasma generator (NASA [122]).] 
Une cathode chaude est protégée par un faible écoulement de gaz inerte. L'écoulement principal du gaz (par exemple l'air) est introduit dans une chambre à l'aval de la cathode. Un constricteur, formé d'une série de disques refroidis par circulation d'eau, permet l'établissement d'un profil d'enthalpie pleinement développé avant l'entrée du plasma dans la tuyère divergente qui lui confère une vitesse supersonique. Le maximum d'enthalpie fournie dans cette configuration est limité par le flux de chaleur que peuvent absorber les parois refroidies $\left(10 \mathrm{~kW} / \mathrm{cm}^{2}\right)$. L'enthalpie moyenne qui peut être obtenue est de $10^{5} \mathrm{~kJ} / \mathrm{kg}$ avec une pression à l'aval du constricteur de 100 à $250 \mathrm{kPa}$ [123]. Les performances de ce type de générateur sont limitées par la tenue des électrodes. Une des dernières versions a permis d'atteindre une enthalpie moyenne de $3 \times 10^{5} \mathrm{~kJ} / \mathrm{kg}$ à $100 \mathrm{kPa}$ et de $3,5 \times 10^{4} \mathrm{~kJ} / \mathrm{kg}$ à $700 \mathrm{kPa}$. La puissance fournie est de $2 \mathrm{MW}$ [124].

\subsection{GÉNÉRATEURS STABILISÉS PAR PAROI SEGMENTÉE} FROIDE ET TRANSPIRANTE. - La figure 49 représente une configuration d'un générateur à plasma utilisé par l'AEDC [125] et produisant une enthalpie de l'ordre de $9 \times 10^{5} \mathrm{~kJ} / \mathrm{kg}$ avec une puissance d'alimentation de $20 \mathrm{MW}$. Les contraintes de refroidissement ne permettent qu'une durée d'opération d'une seconde environ [126]. La performance optimale pouvant être atteinte est de $2 \times 10^{7} \mathrm{~kJ} / \mathrm{kg}$ avec une pression d'environ $1,5 \times 10^{4} \mathrm{kPa}$ [127]. L'augmentation du niveau de pression dans le générateur est accompagnée d'une augmentation sensible des pertes par rayonnement et donc d'un transfert de chaleur plus important aux parois.

4.3 GÉNÉRATEURS STABILISÉS PAR CHAMP MAGNÉTIQUE. - Les pertes par rayonnement, excessives dès lors que la pression augmente, peuvent être limitées si l'on établit un profil de température plat dans le constricteur. Ceci est assuré soit par une décharge R.F. superposée à l'arc [128] créant un chauffage préférentiel des bords de l'arc, soit par un champ magnétique introduisant des turbulences. La figure 50 nous donne le principe de cette configuration utilisée par [129].

Par ailleurs le SNIAS [130] utilise ce même principe de champ magnétique entourant les électrodes et assurant ainsi une rotation forcée du pied de l'arc et une durée de vie plus longue dans deux types de générateurs à plasma : J.P. 50 et J.P. 200 alimentés en courant continu (Fig. 51).

Le J.P. 50 permet de fonctionner à des puissances de l'ordre de 4,8 MW en courant continu. Les électrodes cylindriques et la chambre sont en cuivre refroidi par circulation d'eau forcée. Le débit d'air nominal est de $0,23 \mathrm{~kg} / \mathrm{s}$ et la durée maximum de tir atteint $3 \mathrm{~min}$. avec une cadence de tir de l'ordre de $3 \mathrm{~h}$. Le plasma ainsi obtenu contient peu d'impuretés dues à l'usure.

Quant au J.P. 200, il fonctionne à des puissances de l'ordre de $20 \mathrm{MW}$; il est constitué de quatre ensembles identiques montés en parallèle et débitant simul-

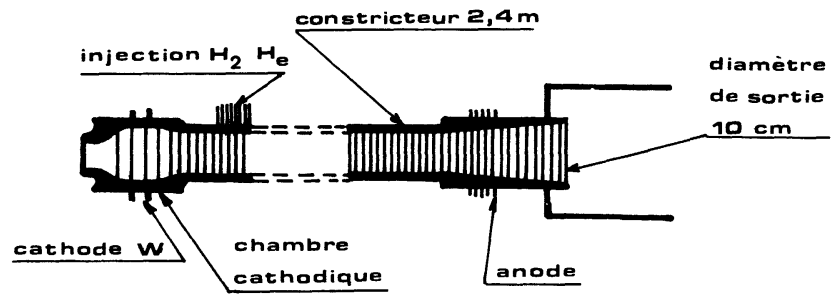

Fig. 49. - Générateur stabilisé par paroi segmentée froide et transpirante AEDC $(P=20 \mathrm{MW})$ [125].

[Schematic of a $20 \mathrm{MW}$ plasma generator stabilized by segmented, cold and transpiration walls (AEDC [125]).]

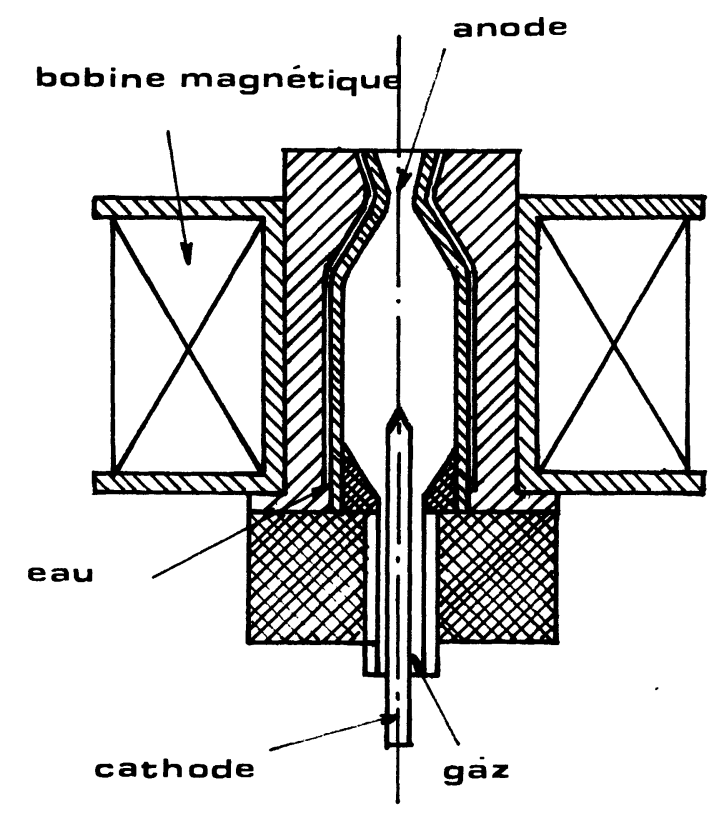

Fig. 50. - Générateur à forte enthalpie stabilisé par champ magnétique [129].

[High enthalpy plasma generator with magnetic field stabilization [129].]



Fig. 51. - Générateur à forte enthalpie à champ magnétique utilisé par la SNIAS $(P=4.8 \mathrm{MW})[130]$.

[High enthalpy plasma generator with magnetic field stabilization $(P=4.8 \mathrm{MW})($ SNIAS $[130])$.]

tanément dans une chambre collectrice. Les électrodes sont en cuivre refroidies par eau. L'amorçage s'effectue par une électrode starter rétractable. L'augmentation 
de la tension et donc de la puissance se fait par augmentation du débit tangentiel et donc le renforcement du vortex obtenu. Le débit d'air dans ce générateur est de l'ordre de $1.5 \mathrm{~kg} / \mathrm{s}$ et la pression peut atteindre 50 bars. La durée maximum du tir est de $1 \mathrm{~min}$. arec une cadence de $4 \mathrm{~h}$. Ces différents types de générateurs en fonctionnement à la SNIAS sont utilisés principalement pour la caractérisation des matériaux ablatables équipant les engins effectuant une réentrée atmosphérique.

4.4 GÉNÉRATEURS STABILISÉS PAR VORTEX. - Le fonctionnement à pression élevée et à un niveau d'enthalpie relativement élevé peut être assuré par une stabilisation de l'arc par un puissant vortex confinant. Le principe de ces générateurs utilisés par la LINDE [131] est présenté sur la figure 52 avec une tuyère supersonique. Les caractéristiques de ce type de générateurs sont résumées sur la figure 53 .

La chute de tension peut atteindre $15 \mathrm{~kW}$, avec des puissances de $50 \mathrm{MW}$. Ces générateurs peuvent, par exemple, chauffer un débit d'air de $3,6 \mathrm{~kg} / \mathrm{s}$ à des enthalpies de l'ordre de $1,5 \times 10^{4} \mathrm{~kJ} / \mathrm{kg}$ et sous une pression de $1,25 \times 10^{4} \mathrm{kPa}$ en utilisant une puissance de $37 \mathrm{MW}$.

Dans le cas des générateurs longs (quelques mètres), un vortex simple peut entraîner une perte de stabilité, on y pallie en introduisant un gaz additif tangentiellement le long du tube de confinement.

L'arc dans ce type de générateur à vortex est toujours entièrement entouré par les électrodes, le rendement est influencé fortement par le transfert de chaleur aux électrodes. Une étude de ce transfert de chaleur à l'électrode est présentée par [132] pour une puissance maximum de $12 \mathrm{MW}$ avec des intensités d'arc de $800 \mathrm{~A}$ et des pressions jusqu'à $2 \times 10^{4} \mathrm{kPa}$. Les pertes électriques dans ces générateurs augmentent linéairement avec l'intensité et avec le carré de la pression. Ainsi, le transfert de chaleur par rayonnement devient le mécanisme prédominant au voisinage de la pression de $2 \times 10^{4} \mathrm{kPa}$.

4.5 GÉNÉRATEUR STABILISÉ PAR COMBINAISON D'UN VORTEX ET D'UN CHAMP MAGNÉTIQUE. - Enfin une combinaison d'un champ magnétique et d'un vortex permet d'assurer un profil de température plat. La masse totale du gaz passant à travers le générateur atteint une température uniforme pour un niveau d'enthalpie déterminé. Un schéma appliquant cette approche est présenté sur la figure 54 d'après [133]. L'arc est forcé de suivre un trajet hélicoïdal par l'action du champ magnétique sur le vortex, ce qui a pour effet d'agrandir le diamètre de l'arc et de produire une distribution uniforme de la température très peu sensible à l'intensité et à la pression.

5. Conclusion. - Cette revue générale des divers générateurs à plasma à faible et forte enthalpies nous montre bien l'étendue de cette activité internationale dans le domaine du plasma thermique. Elle nous permet de percevoir le développement actuel d'un

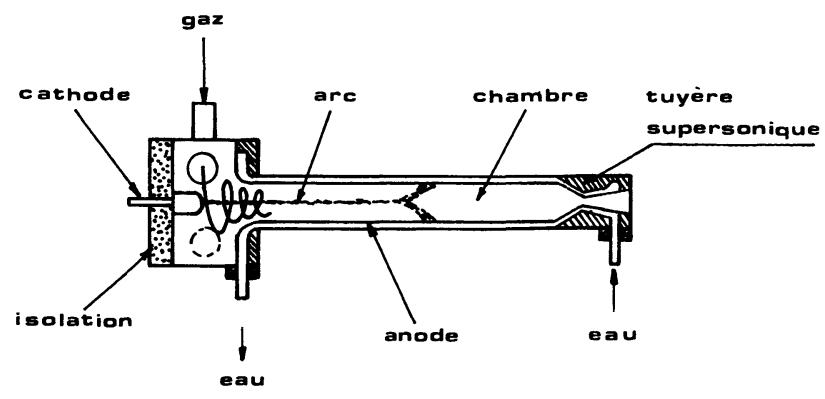

Fig. 52. - Générateur à plasma de forte enthalpie stabilisé par vortex (LINDE [131]).

[Vortex-stabilized high enthalpy plasma generator (LINDE [131]).]

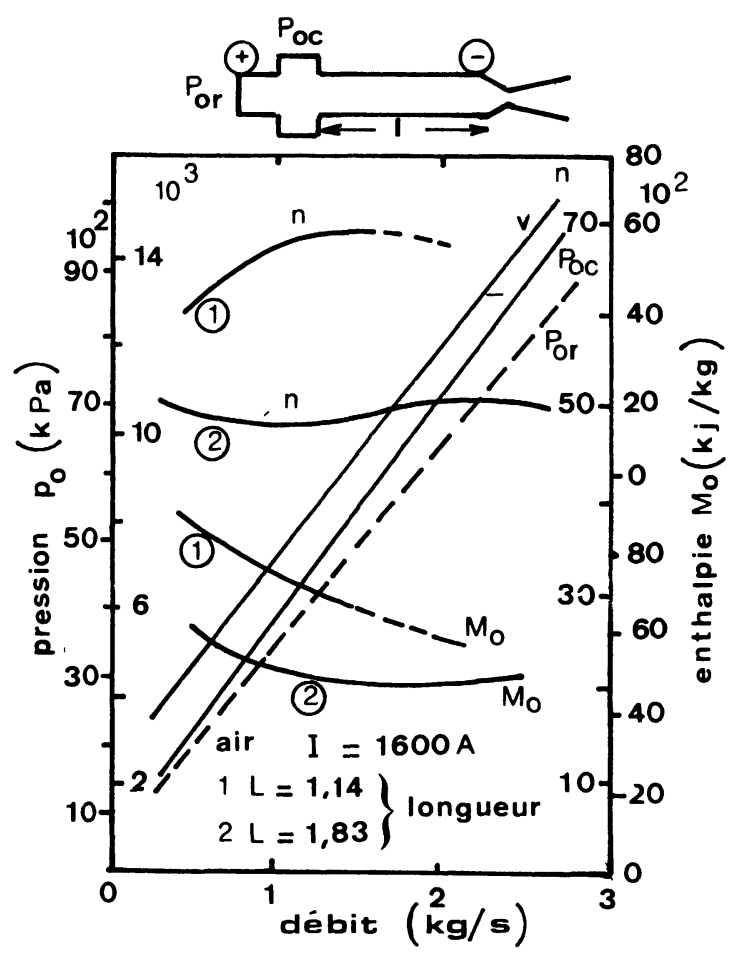

Fig. 53. - Caractéristiques d'un générateur à plasma à vortex [131]. [Performance characteristics of a vortex stabilized plasma generator [131].]

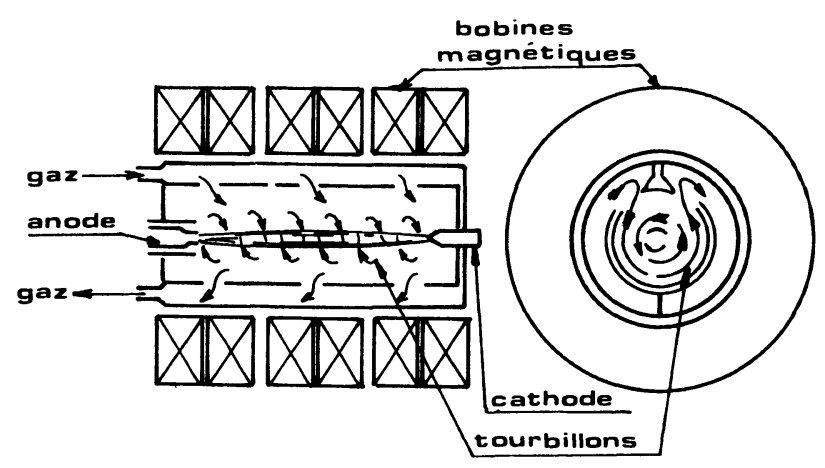

Fig. 54. - Générateur à plasma stabilisé par combinaison d'un vortex et d'un champ magnétique [133].

[Schematic of a vortex stabilized arc plasma generator with superimposed axial magnetic field [133].] 
outil fiable, efficace et utilisable industriellement. Un nombre encore réduit d'applications est maintenant passé au stade industriel, essentiellement d'ailleurs pour des applications spécifiques où la technique plasma est sans concurrence. Cependant on voit, dès maintenant, certaines techniques plasma se substituer aux techniques classiques soit parce qu'elles sont plus simples et moins onéreuses, soit parce qu'elles sont moins polluantes.

Deux raisons principales font accélérer ce développement des utilisations industrielles : la première est l'augmentation considérable et continue du prix des produits pétroliers avec en parallèle la compétitivité en terme de coûts et de disponibilité de l'énergie électrique du fait du développement de l'énergie nucléaire en France notamment. Ces facteurs économiques amènent nécessairement à envisager un certain nombre de problèmes sous un angle nouveau.

La seconde raison est due aux progrès considérables effectués ces dernières années dans le domaine de l'utilisation des plasmas tant au plan des mesures qu'à celui de la compréhension et de la modélisation des phénomènes. Ces progrès ont permis une amélioration sensible des traitements physiques ou chimiques en plasma notamment en ce qui concerne les taux de conversion et les coûts énergétiques des procédés, rendant ainsi ces procédés plasmas concurrentiels, voire supérieurs aux procédés classiques.

Toutefois ce développement de l'utilisation des générateurs à plasima passe obligatoirement :

- par la mise au point de générateurs de forte puissance capables de fonctionner avec des gaz oxydants ou réducteurs ;

- par la poursuite de la modélisation des générateurs pour mieux adapter leurs conditions de fonctionnement (cartographies de températures, des vitesses d'écoulement, des densités d'espèces...) aux problèmes physiques et chimiques à résoudre.

\section{Bibliographie}

[1] Luneau, J., Hancy, H. P., Institut Franco-Allemand de St-Louis. N.T. T54/69 (23.12.1969).

Balageas, D., Mesure des densités de flux par des maquettes montées en dard au générateur de plasma CH10, N.T. ONERA (1969).

Brulard, J., Crabol, J., Lengelle, G. et Verdier, C., Phénomènes physico-chimiques de surface dans l'ablation de plastiques renforcés, ONERA, NT no 195 (1972).

RAEZER et al., Application of DC plasma Arc Heating to Hypersonic propulsion testing. J. Space cr. Rockets 1 (1964) 2 .

[2] HIRSH, M., Décharges $H F$ et microondes Séminaire Chimie des Plasmas, Dourdan 20-24/02/78, ed. A.D.E.R.P. Orsay.

Fehsenfeld, F. C., Evenson, K. M., Broida, H. P., Rev. Sci. Instrum. 36 (1955) 294.

[3] Hubert, J., Moisan, M., Ricard, A., Spectrochim. Acta 33 (1979) 1.

[4] EcKert, H. U., High Temp. Sci. 6 (1974) 99.

[5] Fauchais, P., Bourdin, E., Coudert, J. F., Mc Pherson, R., " High Pressure Plasmas and their applications to ceramic technology " à paraître dans Topics in Current Chemistry, Ed. Veprek K., Venugopalan M. (Springer Verlag, Berlin Heidelberg New York) 1981

[6] Pfender, E., « Electric Arcs \& Arc Gas Heaters » in Gaseous Eléctronics 1, p. 291, edited by M. N. Hirsch, H. J. Oskam (Academic Press, N.Y.) 1978

[7] Vallbona, G., Bonet, C., Foex, M., « Etude d'une cathode en oxyde réfractaire utilisée comme électrode d'un générateur à plasma " J. Phys. D. 8 (1975) 1185-1 197.

[8] Petrie, T. W. and Pfender, E., " The influence of the cathode tip on temperature and velocity fields in a gas-tungsten arc ». Weld. Res. Suppl. december (1970) 588-596.

[9] Mosley, K., Guile, A. E. and Dring, D., " Some aspects of electrode erosion in arc heaters" J. Electrochem. Soc. 119 (1972) 1316-1320

[10] Guile, A. E., "Arc Electrode Phenomena " Proc. IEE, IEE Rev 118 (1971) 1131-1154.

[11] Guile, A. E., «Erosion of non-refractory cathodes in arc plasma devices " Proc. 4th Int. Symp. on plasma Chem., Zurich (1979).

[12] Kanzawa, A., Prender, E., "Numerical Analysis of the joule heating effect on plasma heat transfer " IEEE Trans. Plasma Sci. PS-6 (1978) 33-42.
[13] Johnson, D., Pfender, E., « Modeling and Measurement of the initial anode heat fluxes in pulsed high-current arcs $\gg$ IEEE Trans. Plasma Sci. PS-7 (1979) 44-48.

[14] Smith, J. L., Pfender, E., « Determination of local anode heat fluxes in high intensity, thermal arcs " IEEE Trans. Power Appar. and Syst. PAS-95 (1976) 704-710.

[15] Dinulescu, H. A., Analysis of the anode boundary layer of a high pressure high current arc $\mathrm{Ph}$. D. Thesis, Univ. of Minnesota (1979)

[16] Heberlein, J. V. and Pfender, E., Investigation of the anode boundary layer of an atmospheric pressure argon " IEEE Trans. Plasma Sci.PS-5 (1977) 171-180.

[17] Dinedescu, H. A. and Pfender, E., "Analysis of the anode boundary layer of high intensity arcs "J. Appl. Phys. 51 (1980) 3149-3157.

[18] Pfender, E., "Energy Transport in Thermal Plasmas » Pure Appl. Chem. 52 (1980) 1773-1800.

[19] MAECKER, H., Z. Phys. 158 (1960) 392.

[20] LIU, C. H. and PFENDER, E., Heat Transfer in the anode region of high-intensity arcs Eckert-Festschrift (Hemisphere Publishing Co) 1979.

[21] Knowles, G. R. and Heberlein, J. V., IEEE Trans. Plasma Sci. PS-1 (1973) 15 .

[22] Anderson, J. E. and Eckert, E. R. G., AIAA J. 5 (1967) 699.

[23] Pfender, E., Gruber, G. and Eckert, E. R. G., « Experimental Investigation of a transpiration-cooled, constricted arc ». Proc. Int. Symp. High Temp. Tech. 3rd, I.U.P.A.C. Butterworths, Washington, D. C. (1969) p. 593.

[24] Kugler, T., Miklossy, K., Czechoslovak Patent Specification No 3.097.292 (1963).

[25] Burhorn, F., Maecker, H. and Peters, T. H., Z. Phys. 144 (1956) 244.

[26] BokHaRI, A. and Boulos, M., "Energy balance for a DC plasma torch " Can. J. Chem. Eng. 58 (1980) 171-176.

[27] JoHN, R. R. and BADE, W. L., « Recent Advances in Electric Arc Plasma Generation Technology " ARS Journal January (1961), 4-17.

[28] KubaneK, G. R. and Gauvin, W. H., " Recent Developments in Plasma Jet Technology » Can. J. Chem. Eng. 45 (1967) 251-257.

[29] Fauchais, P., "Etude expérimentale d'un générateur à plasma " Rev. Int. Hautes Temp. Réfract. 5 (1968) 71-87. 
[30] Krichel, R., DruXes, H. and Schmitz, G., Z. Phys. 217 (1968) 336.

[31] Guile, A. E. and NAYLOR, K. A., "Further correlation of experimental data for electric arcs in transverse magnetic fields » Proc. IEEE 115 (1968) 1349-1354.

[32] FauchaIs, P., "Les chalumeaux à plasma à arc électrique " dans Les hautes températures et leurs utilisations en physique et en chimie Tome 1 (Masson \& Cie).

[33] Fey, M. G., Wolf, C. B., HARvey, F. J., Magnetite spheroïdisation using an A.C. arc heater " Int. Round Table on Study and Appl. of Transport Phenomena in Thermal Plasmas. Sept. (1975)

[34] Bonet, C. et al., J. Phys. D. 9 (1976) 141.

[35] Roots, W. K. and KadHim, M. A. H., IEEE Trans. Instrum. Measurements 18 (1969) 150.

[36] Bonet, C., Lamos, C., Foex, M., « Ecoulements de plasma de grande puissance " Entropie, no 36, nov.-déc. (1970).

[37] Schoumaker, H., Four à plasma triphasé. Communication au C.B.E.E. (1971)

[38] KotanchiK, J. N., NASA Langley.

[39] Rykalin, N. N., " Plasma Engineering in Metallurgy and inorganic materials technology ». Pure Appl. Chem. 48 (1976) 179-194.

[40] Zнuкоv, M. F., "Electrode Erosion" in Near electrode processes and electrode erosion in plasmatrons, Zhukov, Novossibirsk, (1977), 123-148 (en russe)

[41] Guile, A. E. and Hiтchсоск, A. H., " The effect of rotating arc velocity on copper cathode erosion "J. Phys. D. 7 (1974) $597-606$

[42] Guile, A. E. and Hiтchсоск, A. H., "The erosion of copper cathodes from vacuum to atmospheric pressure arcs " J. Appl. Phys. 49 (1978) 4275-4276.

[43] GoRsE, C., Contribution au calcul des propriétés de transport des plasmas des mélanges argon-hydrogène et argon-azote. Thèse 3e cycle Limoges (1975).

[44] Bourdin, E., Boulos, M., Fauchais, P., "Conduction heat transfer to a single sphere under plasma conditions " 30th Canadian Chemical Engineering Conference on the National Heat Transfer Symposium, Edmonton 19-22 october (1980), Alberta, Canada.

[45] ShipP, P. and PFender, E., "Controlled generation of submicron metallic Particles in a Plasma Jet " AIChE Symposium Series 75 (1979).

[46] Kassabj, F., Etude de transfert thermique et de l'écoulement dans un réacteur à plasma et contribution à la modélisation d'appareils à gaz plasmagène. Rapport Interne, Laboratoire de Thermodynamique, Université de Limoges (1979).

[47] Bower, W. W. and INCropera, F. P., Heat transfer, Development length and friction factor correlations for the asymptotic region of a laminar arc constrictor $\mathrm{W}$ ärme und Stoffübertragung Bd. 2 (1969) 150-162.

[48] KassabjI, F., Fauchais, P., Heat transfer and flow in a hydrogen plasma reactor J. Physique 40 (1979) C7-321.

[49] HunN, B. D. and Moffat, R. J., "Radiative heat transfer from a plasma in tube flow "Int. J. Heat Mass Transfer 17 (1974) 1319-1328.

[50] Scott, R. K. and Incropera, F. P., « Nonequilibrium flow calculations for the hydrogen constricted arc " AIAA J. 11 (1973) 1714-1719.

[51] SCHMidT, P. S. and LePPERT, G., « Heat transfer from plasma in tube flow » J. Heat Transfer (1970) 483-489.

[52] INCropera, F. P., Bower, W. W., KINGSBURY, R. L., Numerical Studies of Internal, Equilibrium Plasma Flows ASME paper 69, WA/HT-55 (1969).

[53] Kassabj, F., Aubreton, J., Pateyron, B., Fauchais, P., Transfert thermique et écoulement dans les régions de décharge d'un réacteur à plasma à hydrogène Congrès de la Société Française de Physique, Toulouse (1979).

[54] LuKens, L. A. and INCROPERA, F. P., « Electric fields intensity and wall heat transfer measurements for the heating region of an atmospheric cascade arc » Int. J. Heat Mass Transfer 15 (1972) 935-952.
[55] LUKENS, L. A., An experimental investigation of electric field intensity and wall heat transfer for the heating region of a constricted arc plasma $\mathrm{Ph}$. D. Thesis, Purdue Univ. (1971).

[56] Baddour, R. F., Timmins, R. S., The application of plasma to chemical processing Ed. M.I.T. Press (1967).

[57] Fauchais, P., " Utilisation industrielle actuelle et potentielle des plasmas : synthèses, traitement des poudres, traitements métallurgiques, traitements de surface » Revue Phys. Appl. 15 (1980) 1281-1381.

[58] Rykalin, N. N., Kudinov, V. V., " Plasma spraying » Pure Appl. Chem. 48 (1976) 229-239.

[59] Besson, J. L., Vardelle, M., Boch, P., Ind. Céram. (1979).

[60] WILKS, P. H. and THORPE, M. L., "The heating of solids in high temperature plasma " Paper presented at American Chemical Society, Symposium on high temperature, Chicago (1970) 13.

[61] Evans, A. M., Williamson, J. P. H., J. Mater. Sci. 14 (1979) 680.

[62] Mathieu, A. C., Contribution à l'étude expérimentale et théorique de la sphéroidisation de particules d'argiles réfractaires dans un four à plasma Thèse $3^{\mathrm{e}}$ cycle, Limoges (1979).

[63] SCHNell, C. R., et al., " The industrial application of plasma technology for the production of fumed silica "Symposium on Commercial Potential for Arcs and Plasma Processes, Atlantic City, N. J., 8-11 septembre (1974).

[64] Land, U. et al., "Production of smouth and spheroidal ferrosilicon particles » S.A. Pat. 720850, appl. (1971)

[65] WALDIE, B., " Effects of increasing pressure on transfered and non-transfered arcs from a plasma cutting torch " 4 Symposium International de Chimie des Plasmas Zurich (1979) p. 311.

[66] SCHOUmakeR, H. R. P., "four à chauffage plasma " dans Conference proceedings of International Round Table on Study and Application of Transport Phenomena in Thermal Plasma Laboratoire des Ultra-Réfractaires du C.N.R.S. Odeillo sept. (1975).

[67] Sayce, I. G., Pure Appl. Chem. 4, (1976) 215.

[68] Korman, S., Sheer, C., Electrochem. Soc. Symp. Philadelphia (1966).

[69] Gladish, H., Chimie Ingenieur Technik 41 (1969) 204.

[70] Magnolo, G., Can. Min. Metall. Bull. 57 (1964) 57.

[71] Fiedler, H., et al., "Results of plasma melting of steel » Fifth International Symposium on Electroslag Remelting and Other Special Melting Process Pittsburgh, (1974).

[72] Borodacey, A. S., et al., Neue Hütte 22 (1977) 604.

[73] Asada, C., et al., "Plasma Induction Heating" Proceedings Third International Symposium on Electroslag Melting Processes, Pittsburgh USA (1971).

[74] Paton, B., et al., "Plasma arc remelting in copper watercooled crystalliser as a new method of improving metal and alloy properties ". Proceedings Third International Symposium on Electroslag Melting Processes, Pittsburgh USA, (1971).

[75] Kinoshito, T., SHINKU 17 (1975) 61 (en japonais).

[76] MAC RAE, D. R., et al., "Ferrovanadium production by plasma carbothermic reduction of vanadium oxide". T. III Third Int. Symp. on Plasma Chemistry IUPAC Limoges, 13-19 juillet (1977).

[77] Fey, M. G., Harvey, F. J., " Plasma heating devices in the electric energy economy " Met. Eng. Q., may (1976) 27-30.

[78] Pickless, C. A., Wang, S. S., Mc Lean, A., Alcock, C. B., Segsworth, P. S., IEEE Trans. 18 (1978) 369.

[79] Kassabi, F., Pateyron, B., Aureton, J., Fauchais, P., Amouroux, J., Morvan, D., " Technical and economical studies for metal production by plasma-Steelmaking application " IVth International Symposium on Plasma Chemistry, Zurich aug. (1979) p. 236.

[80] Kassabj, F., Pateyron, B., Aubreton, J., Boulos, M. Fauchais, $\mathrm{P}$., " Conception d'un four à plasma de $0,7 \mathrm{MW}$ pour la réduction des oxydes de fer " à paraître dans Rev. Hautes Temp. Réfractaires (1981). 
[81] Gold, R. G., Sandall, W. R., Cheplick, R. G., MaC RAE, D. R., « Plasma Reduction of Iron Oxide with hydrogen and natural gas at $100 \mathrm{~kW}$ and one megawatt" International Round Table on Study and application of transport phenomena in thermal plasmas IUPAC, Odeillo 12-16 sept. (1975) Proceedings.

[82] Tylko, J. K., « The in-flight manufacture of irons and steels in an expanded processive plasma reactor ". III Symposium International de Chimie des Plasmas IUPAC Limoges 13-19 juillet (1977), t. III.

[83] Santen, S., « Plasmamelt a new process for hot metal production " Int. Industrial Seminar on Pilot Plant Experiences. Melting and Processing Technology, Seabrook Island 13-15 october (1980), South Carolina USA.

[84] Fey, M. G., Meyer, T. N., Reed, W. H., « An Electric Arc Heater Process to Produce Solar Grade Silicon ». $4^{\mathrm{e}} \mathrm{Sym}$ posium International de Chimie des Plasmas, Zurich (1979) p. 708.

[85] Heberlein, J. V. R., Lowry, T. N., Meyer, T. N., CiliCERTI, D. F., « The production of tetrachlorosilane by sodium at high temperatures in a laboratory scale Experiment $》 4^{\mathrm{e}}$ Symposium International de Chimie des Plasmas, Zurich (1979), p. 716.

[86] Kimblin, S. U., “ Erosion des électrodes et processus d'ionisation entre les électrodes de l’arc dans le vide et à la pression atmosphérique » in Investigations expérimentales des plasmatrons M. F. Joukov, ed. Nauka Novosibirsk (1977).

[87] KASSABJ, F., Contribution à la réalisation d'un générateur à plasma, source de chaleur dans un four rotatif et à l'étude de l'écoulement du plasma produit Thèse $3^{\mathrm{e}}$ cycle, Limoges (1975).

[88] Baronnet, J. M., Coudert, F., Rakowitz, J., Bourdin, E., Fauchais, P., Pekarec, S., Fonctionnement à l'air d'un générateur de plasma à arc soufflé Congrès de la Société Française de Physique, Toulouse (1979).

[89] Leontiev, A., Volshkov, E. P., « Caractéristiques électriques et thermiques d'un plasmatron de haute enthalpie " in Investigations expérimentales des plasmatrons M. F. Joukov (ed. Nauka Novossibirsk) (1977).

[90] Baronnet, J. M., Contribution à l'étude spectroscopique des plasmas d'azote produits par un générateur à arc souffé; application à la chimie des plasmas : synthèse des oxydes de l'azote. Thèse Doctorat d'Etat, Limoges (1978).

[91] Fauchais, P., Etude des propriétés thermodynamiques des plasmas produits par un générateur à arc thèse d'Etat, Poitiers (1968).

[92] VARDelle, A., Contribution à la mesure statistique des vitesses et des températures de surface de particules injectées dans un jet de plasma d'arc. Thèse de $3^{\mathrm{e}}$ cycle, Limoges (1979).

[93] Lesinski, J., Mizera-Lesinska, B., JurewiCz, J. and BoULOS, M. I., « Particle and gas velocity measurements in a D.C. plasma jet ». Proceedings of 88th AIChE National Meeting, Philadelphia, Pennsylvania, June (1980).

[94] VARDelle, M., Etude de la structure des dépôts d'alumine obtenus par projection plasma en fonction des températures et des vitesses des particules au moment de leur impact sur la cible Thèse de $3^{\mathrm{e}}$ cycle, Limoges (1980).

[95] Browning, J. A., " Techniques for producing plasma jets » in Dynamics of conducting gases proceedings of the Third Biennal Gas Dynamics Symposium Northwesthern University Press (1960) pp. 126-138.

[96] Blatmann, H., Delmas, R., Foex, M., Yerouchalmi, D., Four rotatif de fusion Brevet 95689 - CEA France.

[97] Kassabj, F., Fauchais, P., " Conditions d'amorçage de la superposition de puissance entre deux générateurs à plasma » Revue Phys. Appl. 12 (1977) 1127.

[98] Dallaire, S., Contribution à l'étude d'un four rotatif électrique à électrodes gazeuses. Thèse docteur-ingénieur, Lille (1976)

[99] Aubreton, J., Dallaire, S., Yerouchalmi, D., Adaptation d'un four tournant, chauffé axialement par un plasma d'arcs transférés, à la fusion des métaux et alliages réfractaires " Colloque du Comité Français d'Electrothermie, Versailles 2-3 mai (1974).
[100] HowIE, F. H., SAYCE, I. G., « Plasma heating of refractory melts » Rev. Int. Hautes Temp. Réfract. 11 (1974) 169-176.

[101] Joukov, M. F., Production de plasma à basse température (en russe) Séminaire Franco-Soviétique, Moscou (1979).

[102] Joukov, M. F., Basical calculations of plasmatrons (en russe) Novossibirsk (1979).

[103] JACQUelin, M. H., Réalisation et étude d'un écoulement continu de plasma dans une tuyère en présence d'un champ magnétique axial PST-Air No 391, ed. SDITA, Paris.

[104] Boldman, D. R., Shepard, C. E., FAKan, J. C., Electrode configurations for a windtunnel heater incorporating the magnetically spun electric arc. NASA TN D-1222 (1962).

[105] COUdert, J. F., Contribution à l'étude de la synthèse des oxydes d'azote par chalumeau à plasma Thèse $3^{\mathrm{e}}$ cycle, Limoges (1978).

[106] BONET, C., « Thermal plasma technology for processing of refractory materials " Pure Appl. Chem. 52 (1980) 17071720.

[107] VAI I BO\A, G., Bonet, C.. Baidi., J. M.. " Etude du comportement de cathodes à base de zircone ". Sémunaire francosoviétique Moscou (1979).

[108] Weatherly, M. H. and Anderson, J. E., " A new highcurrent cathode for operation in reactive gases " Electrochem. Technol. 3 (1965) 80-84.

[109] Gerdien, H. and Lotz, A., Wiss. Veröff. Siemens Kons 2 (1922) 489-496.

[110] Weiss, R., Z. Phys. 138 (1954) 170-182.

[111] Miklossy, K., CSSR patent application $n^{\circ} 7083-63$ and 7084-63.

[112] Gross, B., Gricz, B. and Miklossy, K., Plasma Technology I liffe Books Ltd, London (1968).

[113] Schnell, C. R., Hamblyn, S. M. L., Hengartner, K. and WissLer, M., "The industrial application of plasma technology for the production of fumed silica " Powder Technol. 20 (1978) 15-20.

[114] Fey, M. G., « Electric Arc (Plasma) heaters for the process industries » Ind. Heat. june (1976).

[115] Zhidovich, A. I., Kravchenko, S. K., Yasko, O. I., « Plasma generator with bilateral outflow and electrodes with variable diameter » Inzh. Fiz. Zh. 15 (1968), 204.

[116] Shashkov, A. G., Yasko, O. I., « Application of approximate similarity for correlating arc characteristics » IEEE Trans. Plasma Sci. PS1 (1973) 21-37.

[117] Bonet, C., Lamos, J., Foex, M., « Ecoulements permanents de plasma de grande puissance » Entropie n 34-35 (1970), 36-48.

[118] BoNET, C., «Les générateurs de plasma à arc gainé » High Temperatures - High Pressures 3 (1971) 333-343.

[119] Bonet, C., Vallbona, G., Foex, M., Daguenet, M., DumarGUE, P., « Etude du traitement thermique de réfractaires dans un lit fluidisé par un écoulement de plasma » Rev. Int. Hautes Temp. et Réfract. 11 (1974) 11-24.

[120] Gold, D., Bonet, C., Chauvin, G., Mathieu, A. C., « Spheroïdisation of aluminosilicate particles in a three-phase A.C. plasma furnace " Proc. of 4th Int. Symp. on Plasma Chemistry. Zurich september (1979).

[121] Stine, H. A., "The hyperthermal supersonic aerodynamic tunnel » NASA Proc. Int. Symp. High Temp. Technol. september, Butterworths, Washington D.C. (1964).

[122] Shepard, C. E., Watson, V. R. and Stine, H. A., Evaluation of a constricted-arc supersonic jet NASA TN D-2066 (1964).

[123] Vorreiter, J. W. and Shepard, C. E., « Performances characteristics of the constricted arc supersonic jet " Proc. Heat Transfer Fluid Mech. Ins. (1965) p. 42.

[124] ShePard, C. E., KeINer, D. M. and Vorreiter, J. W., $A$ high enthalpy plasma generator for entry heating simulator NASA TN D-4583 (1968).

[125] RICHTER, R., Ultra-high pressure are heater studies AEDC TR 69-180. Arnold Eng. Develop. Center, Arnold Air Force Station, Tennessee, september (1969).

[126] Shepard, C. E., $A I A A J .10$ (1972) 117. 
[127] RICHTER, R., Ultra-high pressure arc heater studies (phase III) AEDC TR-70-106, Arnold Engineering Develop. Center, Arnold Air Force Station, Tennessee, March (1970).

[128] Schreiber, P. W., Hunter, A. M. and Benadetto, K. R., IEEE Trans. Plasma Sci. PE-1 (1973) 60.

[129] Peters, Th., Arc heaters producing flat temperature distributions AGAR Dograph 84, part. 2, (1964), 883.

[130] Allard, M. A., Présentation des moyens d'essais thermiques Comité Français d'Electrothermie, Journées Electroindustrielles, Bordeaux, mai (1978).
[131] EsChenbach, R. C. and SKINNER, G. M., Development of stable high power, high pressure arc air heaters for a hypersonic wind tunnel WADD-TR-61-100 Linde CO, Indianapolis, Indiana, july (1961).

[132] PainieR, J. H., "High pressure arc heater electrode heat transfer study " AIAA/ASME Thermophys. heat transfer conf. july 15, AIAA paper no 74-731 (1974).

[133] CANN, G. L., An Experimental Investigation of a vortex stabilized arc in an axial magnetic field ARL 73-0043 (1973). 